\title{
Review Article \\ The Chemistry of Bioconjugation in Nanoparticles-Based Drug Delivery System
}

\author{
Karolina Werengowska-Ciećwierz, ${ }^{1}$ Marek Wiśniewski, ${ }^{1,2}$ \\ Artur P. Terzyk, ${ }^{1}$ and Sylwester Furmaniak ${ }^{1}$ \\ ${ }^{1}$ Physicochemistry of Carbon Materials Research Group, Faculty of Chemistry, Nicolaus Copernicus University in Toruń, \\ Gagarin Street 7, 87-100 Torun, Poland \\ ${ }^{2}$ INVEST-TECH R\&D Center, 32-34 Plaska Street, 87-100 Toruń, Poland
}

Correspondence should be addressed to Sylwester Furmaniak; sf@chem.umk.pl

Received 10 November 2014; Accepted 1 February 2015

Academic Editor: Mohindar S. Seehra

Copyright (c) 2015 Karolina Werengowska-Ciećwierz et al. This is an open access article distributed under the Creative Commons Attribution License, which permits unrestricted use, distribution, and reproduction in any medium, provided the original work is properly cited.

\begin{abstract}
Nanomedicine is, generally, the application of nanotechnology to medicine. The term nanomedicine includes monitoring, construction of novel drug delivery systems, and any possible future applications of nanotechnology and nanovaccinology. In this review, the most important ligand-nanocarrier and drug-nanocarrier bioconjugations are described. The detailed characterizations of covalently formed bonds between targeted ligand and nanocarrier, including amide, thioether, disulfide, acetyl-hydrazone and polycyclic groups, are described. Also, the coupling of small elements and heteroatoms in the form of R-X-R the "click chemistry" groups is shown. Physical adsorption and chemical bonding of drug to nanocarrier surface involving drug on the internal or external surfaces of nanocarriers are described throughout possibility of the formation of the above-mentioned functionalities. Moreover, the most popular nanostructures (liposomes, micelles, polymeric nanoparticles, dendrimers, carbon nanotubes, and nanohorns) are characterized as nanocarriers. Building of modern drug carrier is a new method which could be effectively applied in targeted anticancer therapy.
\end{abstract}

\section{Introduction: Nanotechnology and Nanomedicine}

Nanotechnology has led to a junction of different fields including chemistry, biology, applied physics, optics, computational analysis and modeling, and materials science. Recent advances in the physical sciences have provided the ability to analyze and manipulate structures at nanometer scales, and this has been accompanied by advances in molecular modeling and computational science. Using them one can predict by modeling and simulation the behavior of biological structures in (the most important for human) aqueous solution [1]. The purpose of nanomedicine is the same as that of medicine: to diagnose as accurately and early as possible and to treat as effectively as possible. It means that side effects should be avoided [2]. Human health-care nanotechnology research can definitely result in immense health benefits [3].
Therefore, there is increasing funding for nanotechnology all over the world [4]. Estimates of the impact from advances emerging from nanotechnology developments over the next 15 to 20 years have been estimated to be approximately $\$ 1$ trillion by studies conducted at the National Science Foundation [3].

Generally, nanomedicine may be defined as the monitoring, reparation, construction, and control of human biologi$\mathrm{cal}$ systems at the molecular level. To do this nanodevices and nanostructures are applied [3]. The prefix "nano-" means the use of materials of which at least one of their dimensions is in the scale range $1-100 \mathrm{~nm}$ [5].

There are numerous methods for drugs delivering into organisms. They include oral, transdermal, transepithelial, and intravenous delivery. All of these methods could be fulfilled using nanostructures as drug containers. Different forms of drug nanocontainers as polymeric nanoparticles, 

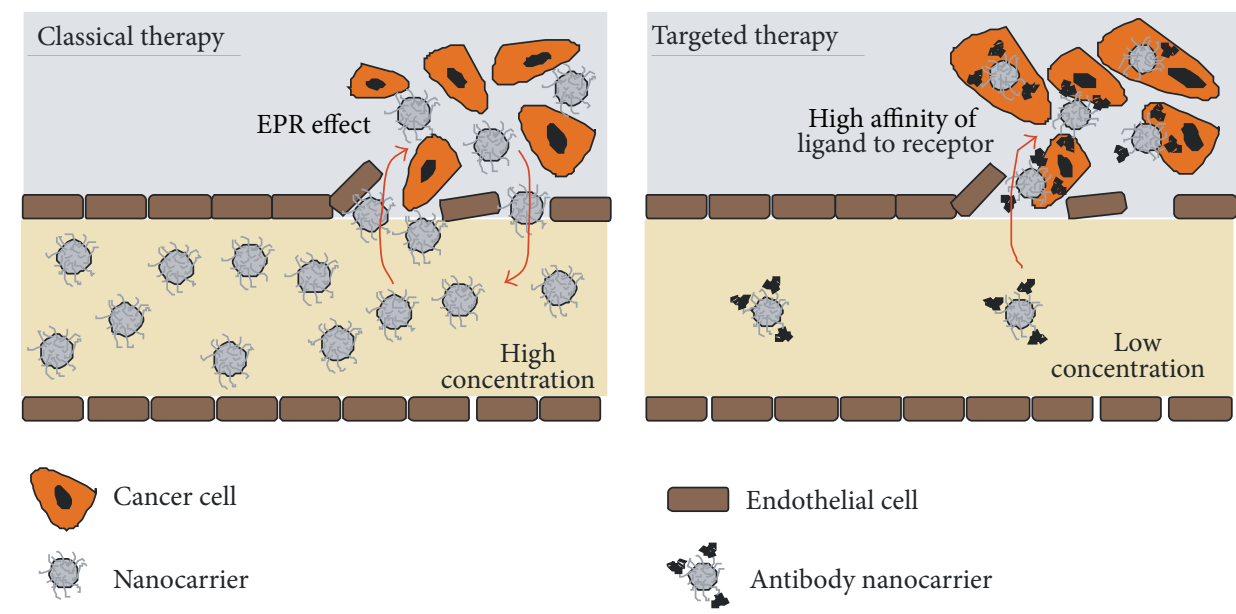

FIGURE 1: The mechanism of drug delivery in classical and targeted therapy (the figure is based on [12, 199]).

liposomes, magnetic nanoparticles, and carbon nanostructures are extensively studied [4-10]. All these DDSs have been reported to have huge biomedical potential.

Nanomedicine is a rapidly developing research area in biological and medical science and the development of nanomedicine research requires collaboration among different scientists: physicians, engineers, molecular biologists, material scientists, chemists, and so forth [8]. Applications of nanotechnology in medicine are especially promising and areas such as disease diagnosis, drug delivery targeted at specific sites in the body (see the next paragraph), and molecular imaging are intensively investigated and some products are undergoing clinical trials. Nanotechnology plays an important role in therapies of the future as "nanomedicine" by lowering doses required for efficacy as well as increasing the therapeutic indices and safety profiles of new therapeutics [9]. The therapeutics are delivery systems in the nanometer size range containing encapsulated, dispersed, adsorbed, or conjugated drugs and imaging agents. Selected nanoscale systems including liposomes, micelles, nanoemulsions, nanoparticulate systems (drug nanoparticles, polymer-, lipid-, carbon-, and ceramic-based, albumin, and nanogels), and dendrimers are used for drug delivery and imaging [9].

Within the next 5 to 10 years, nanomedicine will address many important medical problems by using nanoscalestructured materials and simple nanodevices that can be manufactured today. Many approaches to nanomedicine being carried out today are already close enough to fruition that it is fair to say that their successful development is almost inevitable and their subsequent incorporation into valuable medical diagnostics or clinical therapeutics is highly likely and may occur very soon. Among them, Freitas Jr. [4] mentioned immunoisolation, gated nanosieves, ultrafast DNA sequencing, fullerene-based pharmaceuticals, nanoshells, single-virus detectors, tectodendrimers, radiocontrolled biomolecules, and biologic robots [4]. Flynn and Wei [10] mentioned also the importance of commercialization of nanomedicine.
In the next chapter, we present short review on one of the practical applications of nanotechnology in medicine. We focus on the application of nanoparticles as DDS.

\section{Targeted Anticancer Therapy}

A deeper understanding of the molecular events leading to tumor formation, invasion, angiogenesis, and metastasis has provided a new mechanistic basis for drug discovery: targeted anticancer therapy. By specifically blocking the molecular pathways implicated in the pathogenesis of cancer, targeted anticancer agents are expected to alter the natural course of the disease and, at the same time, to offer an enhanced therapeutic index over traditional cytotoxic agents.

Targeted anticancer therapy must fulfill few requests. Anticancer drugs should be delivered to the cancer cells with minimal activity loss. The drug should kill selectively the cancer cells. The release of drug active form must be, of course, controlled [11]. Simultaneously, only the minimal doses of chemotherapeutic agent should be administered during the targeted therapy (doses should be smaller than those in the traditional chemotherapy). The therapy should also minimize different possible side effects $[11,12]$.

Currently, drugs are usually delivered inside or outside of nanocarriers via the passive or selective mechanism in conventional or targeted therapy, respectively [12] (Figure 1). The traditional mechanism is connected with a drug aggregation process (in form of carrier drug) inside cancer tissues via enhanced permeability and retention effect (EPR) [1315]. This method is based on the abnormal structure of blood vessels near tumor. Thus, the drug reaches easily the tissues near cancer cells $[9,14]$. The most popular drugs used during conventional treatment are doxorubicin [16], paclitaxel [17], methotrexate [18], hexamethylmelamine [19], and gemcitabine [20] and drugs based on platinum [21] like cisplatin (DDP) or carboplatin (Figure 2). The linking of drugs to nanocarriers is described in this paper, together with progressive activity after linking with antibody. 


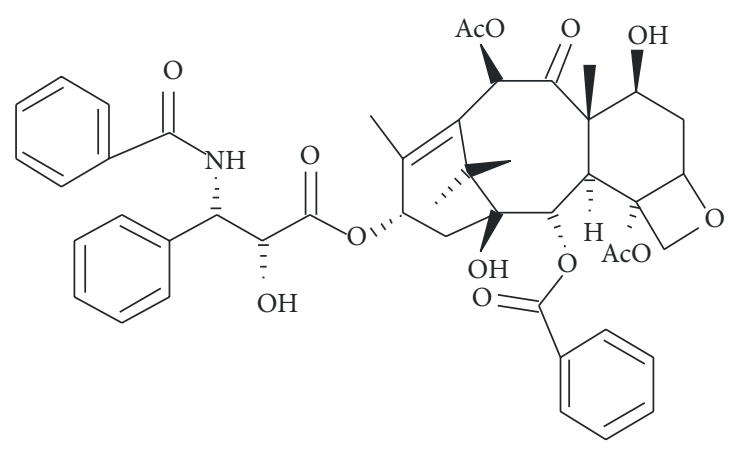

(a)<smiles>CN(Cc1cnc2nc(N)nc(N)c2n1)c1ccc(C(=O)NC(CCC(=O)O)C(=O)O)cc1</smiles>

(c)

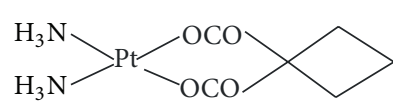

(e)<smiles>N[PH](N)Cl</smiles>

(f)<smiles>COc1cccc2c1C(=O)c1c(O)c3c(c(O)c1C2=O)C[C@@](O)(C(=O)CO)C[C@@H]3OC1CC(N)C(O)C(C)O1</smiles>

(b)<smiles>Nc1ccn([C@@H]2O[C@H](CO)[C@@H](O)C2(F)F)c(=O)n1</smiles>

(d)<smiles>CN(C)c1nc(N(C)C)nc(N(C)C)n1</smiles>

(g)

Figure 2: Chemical structures of drugs: (a) paclitaxel, (b) doxorubicin, (c) methotrexate, (d) gemcitabine, (e) carboplatin, (f) cisplatin, and (g) hexamethylmelamine.

The targeted anticancer therapy uses systems composed of the ligands connected to the carriers of chemotherapeutic agent. Thus, constructed structure facilitates the bioconjugation with appropriate receptors of cancer cells. The targeted anticancer therapy, in contrast to the conventional treatment, supports the overexpression of cancer cell receptors and the affinity of ligand to receptor $[12,14,15]$. The main advantage of targeted treatment is the delivery of chemotherapeutic agents to the most resistant cancer cells and longtime circulation inside them. Application of this method guarantees high concentration of drug inside the tumor. Moreover, drug cannot be released back to the blood. The main factor determining the type of used ligand is immunogenicity [13].

\section{Nanoparticles as Drug Delivery System}

3.1. The Importance of Proper Drug Delivery: The Role of Nanoparticles. The major practical purpose of nanotechnology in medicine is the application of nanoparticles in DDS [22]. The reason of this is that during the past two decades researchers involved in the development of pharmaceuticals have understood that drug delivery is a fundamental part of drug development, and a wide range of DDS has thus been designed [3]. It is also very important that, at present, 95\% of all new potential therapeutics have poor pharmacokinetics and biopharmaceutical properties [9]. The therapeutic index of nearly all drugs currently being used would be improved if they were more efficiently delivered to their biological targets through appropriate application of nanotechnologies. Some drugs that have previously failed clinical trials might also be reexamined using nanotechnological approaches [22].

What are the requirements for an effective and safe drug? For example, in anticancer therapy there must be an adequate drug concentration in the body to allow for an effective dose at the tumor site. The target must be strongly inhibited, with the function essential for tumor cell viability. The drug must have a high toxicity toward the tumor or a favorable therapeutic window. Many authors have delivered a variety of drugs such as hydrophilic and hydrophobic drugs, proteins, vaccines, and biological macromolecules using nanoparticles 
as carriers. For the delivery of antigens for vaccination [3], three main types of gene delivery systems have been described: viral vectors, nonviral vectors (in the form of particles such as nanoparticles, liposomes, or dendrimers), and the direct injection of genetic materials into tissues using so-called gene guns [3]. Nanostructured architectures are promising candidates that will enable targeted delivery of novel drug compounds. Nanoscale drug delivery mechanism has effects on continuous drug release and intracellular entry capability. Moreover, it minimizes side effects and allows for the direct treatment of the cause of the disease rather than the symptoms of the illness. Generally nanoparticles [14, 23, 24]

(i) have advantage over larger microparticles, because they are better suited for intravenous delivery,

(ii) have been highly exploited for controlled drug release and site-specific drug targeting,

(iii) have shown promising results in the case of sitespecific drug targeting for treating various diseases including cancer, human immunodeficiency virus infection, and central nervous system disorders,

(iv) have a higher surface to volume ratio as compared with bulk material, and therefore the dose and frequency of administration would be reduced hence increasing patient compliance; recently, it was shown that solid lipid doxorubicin loaded nanoparticles have potential to acne,

(v) have the additional advantage of prolonged circulation in the blood, which would facilitate extravasation and passive targeting (nanoparticles made with hydrophilic polymers),

(vi) avoid opsonization with particle size less than $100 \mathrm{~nm}$ (hydrophilic nanoparticles). These systems prolong the duration of action as well as increasing the targeting of the drug to specific sites [25].

Multifunctional nanodelivery systems could combine targeting, diagnostic, and therapeutic actions. There are already an astonishing number of emerging applications. These purposes either take advantage of the unique properties of nanoparticles as drugs or components of drugs per se or are designed for new approaches to controlled release, drug targeting, and salvage of drugs with low bioavailability [3].

As mentioned above, the success of a therapy depends on the drug delivery method. Its importance is exemplified by the presence of more than 300 companies based on the United States involved with developing drug delivery platforms. In addition to the commonly used oral and injection routes, drugs can also be administered through other means, including transdermal, transmucosal, ocular, pulmonary, and implantation delivery. The mechanisms used to achieve alternative drug delivery typically incorporate one or more of the following materials: biologics, polymers, silicon-based materials, carbon-based materials, or metals [3].

In this paragraph, we will focus mainly on carbon nanomaterials in drug delivery reporting the results of new findings. The applications of biologic structures, polymers, dendrimers, silicon-based structures, and some carbon materials in drug delivery were reviewed in [24]. In [26], the review on nanoshells, carbon nanotubes, dendrimers, superparamagnetic nanoparticles, and liposomes applied in cancer therapeutics is presented. All of these nanotechnology platforms can be multifunctional, and so they are frequently named "smart" or "intelligent." The authors raise awareness of the physiological challenges for the application of these therapeutic nanotechnologies, in light of some recent advances in our understanding of tumor biology [26].

When drugs and imaging agents are associated with nanoscale carriers, their volumes of distribution are reduced. Nanoscale DDS also has the ability to improve the pharmacokinetics and increase biodistribution of therapeutic agents to target organs, which will result in improved efficiency. Drug toxicity is reduced as a consequence of preferential accumulation at target sites and lower concentration in healthy tissues. Nanocarriers have been designed to target tumors and inflammation sites that have permeable vasculature. Targeting and reduced clearance increase therapeutic index and lower the dose required for efficacy. Delivery systems have been shown to increase the stability of a wide variety of therapeutic agents such as small hydrophobic molecules, peptides, and oligonucleotides. Nanocarriers composed of biocompatible materials are investigated as safe alternatives to existing vehicles that may cause hypersensitivity reactions and peripheral neuropathy [9]. A number of additional obstacles can be overcome with various novel applications of nanodrug delivery. Many drugs are not very soluble, making it difficult to administer therapeutic doses. These compounds can be "solubilized" by formulating them into crystalline nanosuspensions that are stabilized by surfactants or by combining them with organic or lipid nanoparticles that keep them in circulation for longer periods. If an efficacious compound has a short half-life in the circulation, its stability can be increased tremendously by encasing it within, for example, nanosized liposome as a drug carrier. In the case of cancers, for example, of central nervous system, many drugs have difficulty in crossing the blood-brain barrier to attack the tumor. Drug-loaded nanoparticles are able to penetrate this barrier and have been shown to greatly increase therapeutic concentrations of anticancer drugs in brain tumors. The best way to increase the efficiency and to reduce the toxicity of a drug is to direct it into its target and maintain its concentration at the site for a sufficient time for therapeutic action to take effect [22]. The majority of solid tumors exhibit a vascular pore cutoff size between 380 and $780 \mathrm{~nm}$ [9], although tumor vasculature organization may differ depending on the tumor type, its growth rate, and microenvironment. Therefore, particles need to be of a size much smaller than the cutoff pore diameter to reach to the target tumor sites. By contrast, normal vasculature is impermeable to drug associated carriers larger than 2 to $4 \mathrm{~nm}$ compared to free, unassociated drug molecules. This nanosized window offers the opportunity to increase drug accumulation and local concentration in target sites such as tumor or inflamed sites by extravasations and significantly to reduce drug distribution and toxicity to normal tissues [9].

Ideal DDS can be achieved by creation of materials undergoing no chemical changes and satisfying the demands 
of biodegradability and biocompatibility of the nanoparticles carrier, the rate of biodegradation of the carrier, and the release dynamics of the drug $[15,27]$. For example, for socalled passive targeting to be successful, the nanocarriers need to circulate in the blood for extended times so that there will be multiple possibilities for the nanocarriers to pass by the target site. Nanoparticulates usually have short circulation half-lives due to natural defense mechanisms of the body to eliminate them after opsonization by the mononuclear phagocytic system (also known as reticuloendothelial system). Therefore, the particle surfaces need to be modified to be "invisible" to opsonization. A hydrophilic polymer such as polyethylene glycol (PEG) is commonly used for this purpose because it has desirable attributes such as low degree of immunogenicity and antigenicity, chemical inertness of the polymer backbone, and availability of the terminal primary hydroxyl groups for derivatization. PEG-grafted liposomes, in the size range of 70 to $200 \mathrm{~nm}$, containing 3 to $7 \mathrm{~mol} \%$ methoxy-PEG-2000 grafted to distearoyl phosphatidylethanolamine (DSPE) or dipalmitoyl phosphatidylethanolamine, showed extended circulation half-lives of 15 to 24 hours in rodents and up to 45 hours in humans, whereas non-PEGylated liposomes had half-lives of 2 hours $[15,27]$.

Nanocarriers typically consist of macromolecular materials with the active principle either dissolved within a polymeric matrix, entrapped inside lipid, encapsulated, or adsorbed onto surfaces of particles. Accordingly, they can be classified into mainly two types: nanocapsules and nanospheres. The former are vesicular systems in which drug molecules are surrounded by a membrane, whereas the latter are matrix systems with the drug molecules dispersing throughout [2]. Though the technology is still young, more than 1000 nanopharmaceutical patents have been issued by the U.S. Patent and Trademark Office (U.S. PTO) during the period 1999-2008 [2]. Nanotechnology-based methods of synthesis are most commonly developed on the basis of two rational designs: either top-down or bottom-up engineering of individual components. The top-down process involves starting with a larger object and breaking it up into nanostructures through etching, grinding, or ball milling. The process can be accelerated by addition of chemicals or using laser. Microscale or macroscale manufacturing, like silicon microfabrication and photolithography, is often accomplished as top-down process. However, the method is time-consuming and frequently generates considerably broader particle size distribution. The bottom-up technique refers to synthesis based on atom-by-atom or molecule-bymolecule arrangement in a controlled manner. The process takes place through controlled chemical reactions, in either gas or liquid phase, resulting in nucleation and growth of nanoparticles. Bottom-up techniques (like supercritical fluid antisolvent techniques, precipitation methods, etc.) create heavily clustered masses of particles that do not break up on reconstitution [2]. All preparation methods: highpressure homogenization, complex coacervation, coprecipitation, salting-out, nanoprecipitation, solvent emulsificationdiffusion, supercritical fluid, rapid expansion of supercritical solutions, supercritical antisolvent precipitation, and self-assembly methods, were in detail described in [2]. Among nanoparticles they describe polymeric nanoparticles, solid lipid nanoparticles, magnetic nanoparticles, metal and inorganic nanoparticles, quantum dots, polymeric micelles (PMs), phospholipid micelles, and colloidal nanoliposomes.

3.2. Drug Delivery Systems. The examples of recent application of nanoscale systems for drug delivery are shown in Figure 3. We focus on solid nanomaterials most often used as nanocarriers. These carriers, after joining some ligands and/or drugs, can be used in designing of the systems for targeted therapies as described in the following sections.

Liposomes and lipids have been used as DDS since 1960. Liposomes are defined as vesicles in which an aqueous volume is entirely surrounded by a phospholipid membrane. Liposome size can vary from $30 \mathrm{~nm}$ up to several micrometers and can be uni- or multilamellar [9]. Recently it was shown that solid lipid doxorubicin loaded nanoparticles have potential to serve as a useful therapeutic approach to overcome the chemoresistance of Adriamycin-resistant breast cancer. Koren et al. [28], Koshkaryev et al. [29], and Etzerodt et al. [30] showed that the entrapment of some chemical compounds inside modified liposomes (resp. by antibody $[28,30]$ and transferring [29]) causes an increase of apoptosis of cancer cells. Moreover, the solid lipid nanoparticle system could be generally applied for the delivery of many chemotherapeutic agents in chemotherapy-resistant cancers [31].

Micelles are self-assemblies of amphiphiles that form supramolecular core-shell structures in the aqueous environment. Hydrophobic interactions are the predominant driving force in the assembly of the amphiphiles in the aqueous medium when their concentrations exceed the critical micelle concentration. Phospholipid, Pluronic, poly(L-amino acid), and polyester micelles are most often applied. In [32] authors summarize advances related to targeted anticancer drug delivery to tumor sites using PMs via active and passive mechanisms (see below). PMs can be conjugated with diverse ligands such as antibodies fragments, epidermal growth factors, $\alpha$-2-glycoprotein, transferrin, and folic acid.

Over the past few decades, there have been repeated attempt to develop an ideal DDS that selectively acts against diseased cells but is not harmful to healthy cells. PMs are one of the nanocarriers that can do this. Also, as most of the anticancer drugs are poorly water-soluble, PMs are convenient drug carriers for carrying as well as targeting such drugs to tumors. Collectively, all these studies suggest that drugs encapsulated in micelles show enhanced therapeutic index in solid tumors, correlating to their passive targeting, taking advantage of tumor characteristics as well as active targeting using various mechanisms and fewer side effects in comparison with conventional drug formulations. Among these, a few PM formulations have been successfully developed and a few more are at preclinical stage. There is a dire need to translate these proven experimental advantageous concepts into clinical practice to diminish the death rate from cancers and increase hope in cancer chemotherapy [32].

Besides micelles, there is the other group of nanomaterials forming self-assembling structure, known as cell-penetrating 


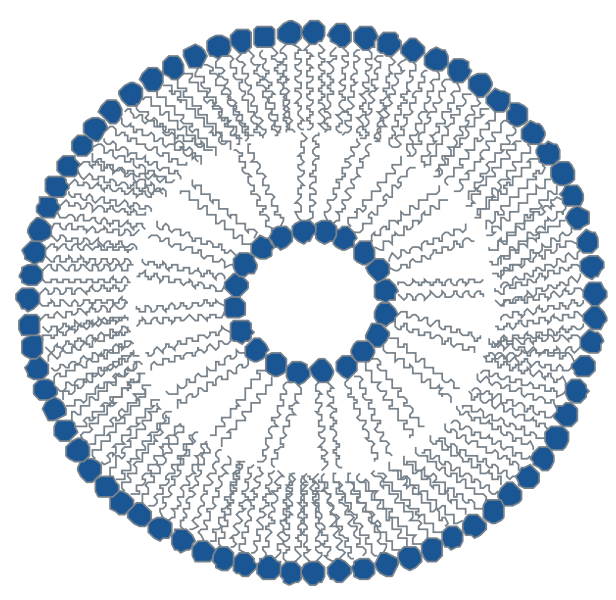

(a)

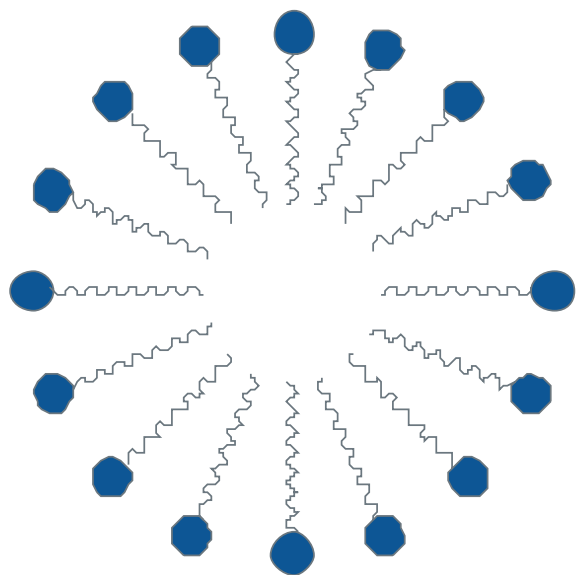

(b)

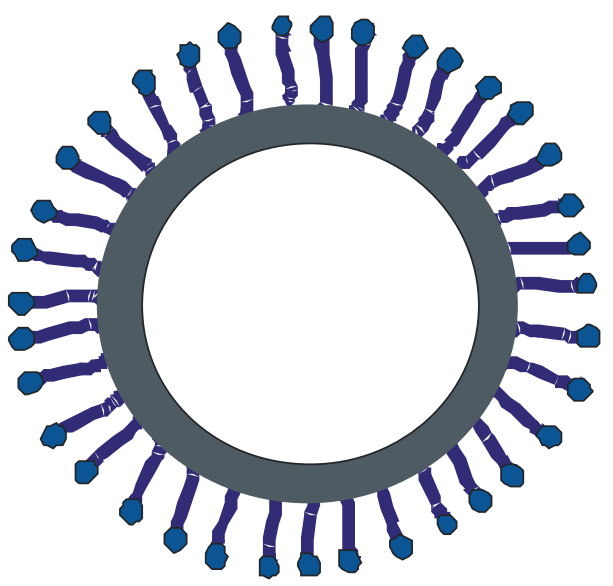

(c)

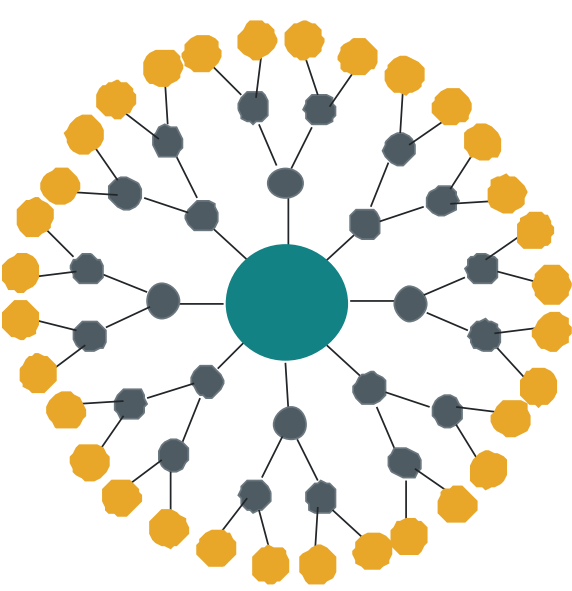

(d)

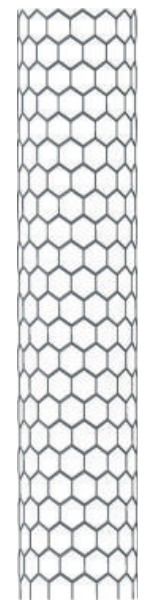

(e)

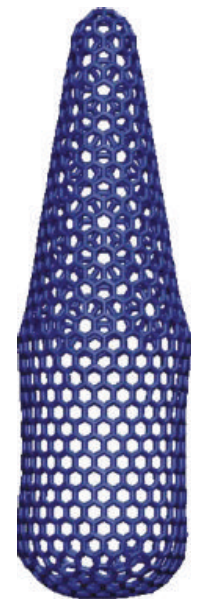

(f)

FIGURE 3: Schematic representation of nanocarriers: (a) liposome, (b) micelle, (c) polymeric nanoparticle, (d) dendrimer, (e) carbon nanotube, and (f) carbon nanohorn.

peptides (CPPs). These peptides could be applied to identify hydrophobic anticancer drugs and intracellular delivery of biomolecules such as nucleic acids (siRNA, pDNA). The application of CPPs in novel delivery systems is favorable because of numerous advantages: biocompatibility, low toxicity, easy preparation, and stability of the structure [33-36]. Arukuusk et al. [33] proved that the addition of hydrophobic moieties to CPPs improves their properties during application as the nucleic acid delivery systems. Deshayes et al. [37] and Hou et al. [38] described the creation of siRNA delivery system based on the CPPs. The process of peptides selfassembly proceeds spontaneously during the contact with siRNA. A stable structure was formed mainly due to both electrostatic and hydrophobic interactions. Those complexes easily penetrate the cell and could be applied to successfully primary cell lines.

Polymeric Nanoparticles. Many methods have been developed for preparing polymeric nanoparticles. These methods can be classified into two main categories according to whether the formulation requires a polymerization reaction or is achieved directly from a macromolecule or preformed polymer. Polymerization methods can be further classified into emulsion and interfacial polymerization, and there are two types of emulsion polymerization: organic and aqueous, depending on the continuous phase. Nanoparticles can be also prepared directly from preformed synthetic or natural polymers and by desolvation of macromolecules. Recently these polymeric systems have been prepared by nebulization techniques. In [39] authors present all these methods including their detailed procedures and technological advantages, as well as providing several examples of encapsulants that are entrapped into or adsorbed to these particles. The evolution of nanoparticle preparation methods has been marked by three aspects: need for less toxic reagents, simplification of the procedure to allow economic scale-up, and optimization to improve yield and entrapment efficiency. Efficient drug entrapment and transition to large scale are of highest importance to industrial applicability. Depending on the physicochemical characteristics of a drug, it is now possible to choose the best method of preparation and the best polymer to achieve an efficient entrapment of the drug. Nevertheless, there are several problems remaining to be solved. The process is not suitable to all drugs. In addition, the 
postpreparative steps, such as purification and preservation, particularly important for nanocapsules, and residual solvent analysis must be extensively investigated. Other difficulties such as the formation of an incomplete or of discontinuous film with inadequate stability of certain active components, no reproducible or predictable release characteristics, causes that the final product is economically unfeasible [39]. In [40] authors discuss possibilities of the polymeric nanoparticlebased technique of targeted drug delivery through the bloodbrain barrier. The biodistribution of novel nanoparticles showed two orders of magnitude greater efficiency in comparison to other known drug carriers [40].

Dendrimers. The role of dendrimers in delivery of different compounds (e.g., 5-fluorouracil, primaquine phosphate, doxorubicin, artemether, tamsulosin, indomethacin, tropicamide, and pilocarpine) is presented in [41]. Authors discuss the methods of intravenous, transdermal, ophthalmic, and oral delivery. There are different results that prove the versatility of dendrimers, and some very important in vitro studies with in vivo potential further endorse this versatility. More detailed studies on the routes already investigated and studies on other routes for dendrimer-mediated drug delivery are required; yet the existing data emphasized the potential of dendrimers as drug carriers via various routes. However, the toxicological status of candidate dendrimers must be established conclusively before drawing any final conclusions in this regard [41]. Interesting review describing dendrimers is presented by Wen et al. [42].

Carbon Nanotubes. Basic properties and application of carbon nanotubes in drug delivery were presented in [43-47]. Different studies [48-54] describe the application of carbon nanotubes in DDS. Firme III and Bandaru [45] describe the most popular strategies applied to increase the solubility of nanotubes. The role of defects is also discussed and finally it is concluded that the lack of centralized toxicity database limits makes the comparison between research results impossible. Jain et al. [55] present a novel cascade of chemical functionalization of multiwalled carbon nanotubes (MWCNTs) through chemical modification by a carbohydrate as Dgalactose. Galactose-conjugated MWCNTs were synthesized involving the sequential steps of carboxylation, acylation, amine modification, and finally galactose conjugation. The modification of MWCNTs with galactose was investigated by different methods at every sequential step of functionalization. Size and surface characteristics of chemically modified MWCNTs were monitored. That galactosylation improved dispersibility of MWCNTs in aqueous solvents was confirmed by investigation of their dispersion characteristics at different $\mathrm{pH}$ values. Thus, the galactosylated MWCNTs could be used for delivery of different bioactive(s) as well as active ligand (galactose) based targeting to hepatic tissue [55].

Carbon nanohorns belong to a new class of carbon materials, similar to carbon nanotubes. Single-walled carbon nanohorn (SWNH) aggregates, composed of thousands of graphitic tubules (similar in structure to single-walled CNTs) having wide diameters of $2-5 \mathrm{~nm}$, have a spherical structure with a diameter of $50-100 \mathrm{~nm}$. On the basis of their morphology, they were classified into dahlia, bud, and seed types. SWNHs contain no metal catalyst because they are produced by laser ablation of a pure graphite target. This means that the effects of metal impurities can be excluded when determining toxic responses, enabling investigation of the pure toxicological effects of nanometer-sized graphitic structures. To avoid potential health hazards caused by occupational exposure to SWNHs and to promote industrial and biomedical applications of SWNHs, the toxicity of SWNHs should be proactively investigated from various aspects. Comprehensively investigated in vivo and in vitro toxicities of as-grown SWNHs lead to conclusions that carbon nanohorns are nontoxic [56].

There are much more important materials that can be used as drug carriers: quantum dots [57, 58], Pluronic [59, 60], mesoporous silica $[61,62]$, nanoemulsions $[63,64]$, drug nanocrystals $[65,66]$, ceramic-based nanoparticles [67], albumin nanoparticles $[68,69]$, nanogels $[70,71]$, magnetic nanoparticles $[72,73]$, and so forth; however, in this review we focus on (in our opinion) the most important ones. Also, proteins are promising delivery agents. They could be bioconjugated with drugs, as albumin-bound paclitaxel, forming Abraxane nanoparticle. The formation of albuminpaclitaxel linking is prepared via homogenization process at high pressure [74]. Abraxane is used as Cremophor ELfree formulation. Thanks to this, the system is more effective and less toxic than conventional drugs. Chemotherapeutic agent is released from Abraxane nanoparticle via the albumin receptor in tumor blood vessel $[74,75]$. Albumin-paclitaxel conjugation was successfully applied against gastric [75], lung [74], and metastatic breast [76] cancer.

\section{Covalent Bond Formation between Targeted Ligand and Nanocarrier}

Various methods have been employed to link ligands with reactive groups of the surface of the nanocarriers, and the methods can be classified into covalent and noncovalent conjugations [77]. Common covalent coupling among the other methods involve conjugation of

(i) 2 thiol groups,

(ii) 2 primary amines,

(iii) a carboxylic acid and primary amine,

(iv) maleimide and thiol,

(v) hydrazide and aldehyde,

(vi) a primary amine and aldehyde.

On the other hand, the noncovalent bonding proceeding by physical association of targeted ligands to the nanocarrier surface has the advantage due to avoiding of rigorous, destructive reaction agents. However, there are some problems, such as weak bonding and low control of reaction. Also, the ligands may not be in the desired orientation after the decoration process [9].

4.1. Amide Group. The formation of amide bond proceeds by two stages. During the first one, carboxylic acid groups 


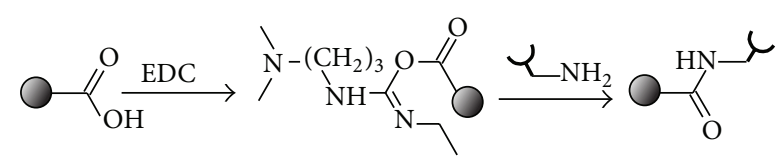

SCHEME 1

on the carrier surface are activated by EDC 1-ethyl-3-(3dimethylaminopropyl) carbodiimide. This reagent is usually applied as carbodiimide which can form different chemical structures [77]. EDC has good solubility in water. This property enables direct application of EDC in aqueous solutions without addition of any organic compounds. These conditions are suitable for the attachment of bioactive molecules to the carrier surface. During the reaction of EDC with carboxylic group, the active form of intermediate product $\mathrm{O}$ acylisourea ester is formed. The latter reacts with primary amine forming amide bond (Scheme 1).

The main advantage of this method is that applied ligand does not need any preliminary modifications usually causing the loss of its activity $[78,79]$. The possibility of activation of carboxylic groups derived from peptide could be some inconvenience in the case of amino acids. These carboxylic groups should have been locked, for example, by NHS (Nhydroxysuccinimide). Ishida et al. [80] presented the addition of transferrin to liposome decorated by polyethylene glycol. They employed DSPE-PEG-COOH system which was the source of required carboxylic acid groups. The product in the form of TF-PEG-liposome has interesting properties: long time of biodistribution and large accumulation in brain tumor. Also, Maruyama [81, 82] or Blume [83] et al. described the formation of amide bond between ligand and liposome surface decorated with PEG-COOH. Zeng's group [84] reported the attachment of EGF with carboxylic group of micelle. The product was employed in the targeted delivery of drug. Also the formation of amide bond formed on the carbon nanotube surface was reported. Ou et al. [85] reported the SWCNTs modification by PEG and antibody (mAb), which was selectively encapsulated by integrin receptor of cancer cells. This process is schematically shown in Scheme 2.

The SWNT-PEG-mAb is characterized by a high stability, low toxicity, and high dispersion in water environment. The product was effectively captured by receptors of cancer cells [85]. Zhang et al. [86] modified oxidized MWCNT surface by biocompatible polyamidoamine dendrimer (PAMAM). The PAMAM chains were successfully conjugated into the surface of carbon nanotubes. This was confirmed by TEM images. The MWCNTs-PAMAM has very good dispersion and stability in aqueous solution. Some extra tests performed by authors confirmed the efficiency of application in gene therapy [86]. Dvir et al. [87] showed the amide conjugation between targeted ligand and liposome. This system during in vitro tests was effectively used against cardiac cells [87]. Chou et al. [88] described the amide conjugation between antibody and functionalized multiwalled carbon nanotubes. This system was applied successfully in targeted photothermal therapy. Authors confirmed the better photothermal effect of the f-MWCNTs connected with antibody for cancer cell destruction.

The above described information was connected with the activation of the carboxylic group on the surface of nanocarrier. Alternative method is associated with the activation of primary amine of nanocarrier as shown in Scheme 3.

This process takes place when homobifunctional dithiobis(succinimidyl propionate) (DSP) is used [77]. The DSP was used in the synthesis of drug carrier which was applied in the targeted breast cancer therapy [89]. The DSP activates amine groups of carriers. Additionally, application of NHS allows the formation of ester during the reaction with monoclonal antibody (trastuzumab). The methodology was very efficient and selective throughout cancer gene therapy.

4.2. Thioether Group. Thioether bond is formed during the reaction between thiol group and $\mathrm{C}_{1}$ carbon of maleimide which is attached to the $\mathrm{R}_{2}$ carrier (see Scheme 4 ). The reaction runs quickly and under mild conditions: at the room temperature and in aqueous solution [12]. Formed thioether bond is stable within 24 hours in human serum even in the presence of reduction agent, for example, DTT [77, 78, 90]. Unfortunately, the selectivity towards the thioether group formation is quite low in aqueous solution, due to the side reactions, such as intermolecular rearrangement or formation of disulfides. The efficiency of reaction could be increased through addition of activating agents, for example, SPDP $\mathrm{N}$-succinimidyl-3-(2 pyridyldithio) propionate or SATA Nsuccinimidyl-S-acetylthioacetate. Application of the systems containing the thioether bonds guarantees the high selectivity of delivery and the long time of distribution [12, 78].

Kirpotin et al. [91] described the synthesis of a selective DDS based on liposome, cholesterol, and PEG modified DSPE. Antibodies were conjugated to the nanocarrier through free thiol group. The synthesis is based on two ideas. First is the coupling of antibody with double layer of liposome. Alternatively, the second provides a simple conjugation of ligand to the distal end of PEG chains. Authors demonstrated that the uptake of anti-HER2-immunoliposome correlated with density of cancer cell surface and with the effect of tumor reduction. The efficiency of the process has been increasing with increasing amount of encapsulated antibody Fab. Similar techniques with the use of different antibodies were applied successfully by, for example, Maruyama et al. [81], Allen et al. [92], Hansen et al. [93], Zalipsky et al. [94], Park et al. [95], and Ren et al. [96]. Produced systems were effectively used in drug delivery, especially for doxorubicin.

Anhorn et al. [97] were pioneers in the synthesis of DDS based on monoclonal antibody trastuzumab and nanoparticle doped with doxorubicin. The conjugation had a nature of thioether bond. The covalent bonding took place between thiol group of ligand and maleimide fragment of a carrier (Scheme 5). Initially, the carrier was modified by molecules of doxorubicin. Nanoparticles were activated through poly(ethylene glycol)-R-maleimide- $\omega$-NHS ester. Applied ligand enabled effective uptake of anticancer system by HER2 receptor of breast cancer cells. The drug is characterized by a long time of circulation in blood without 

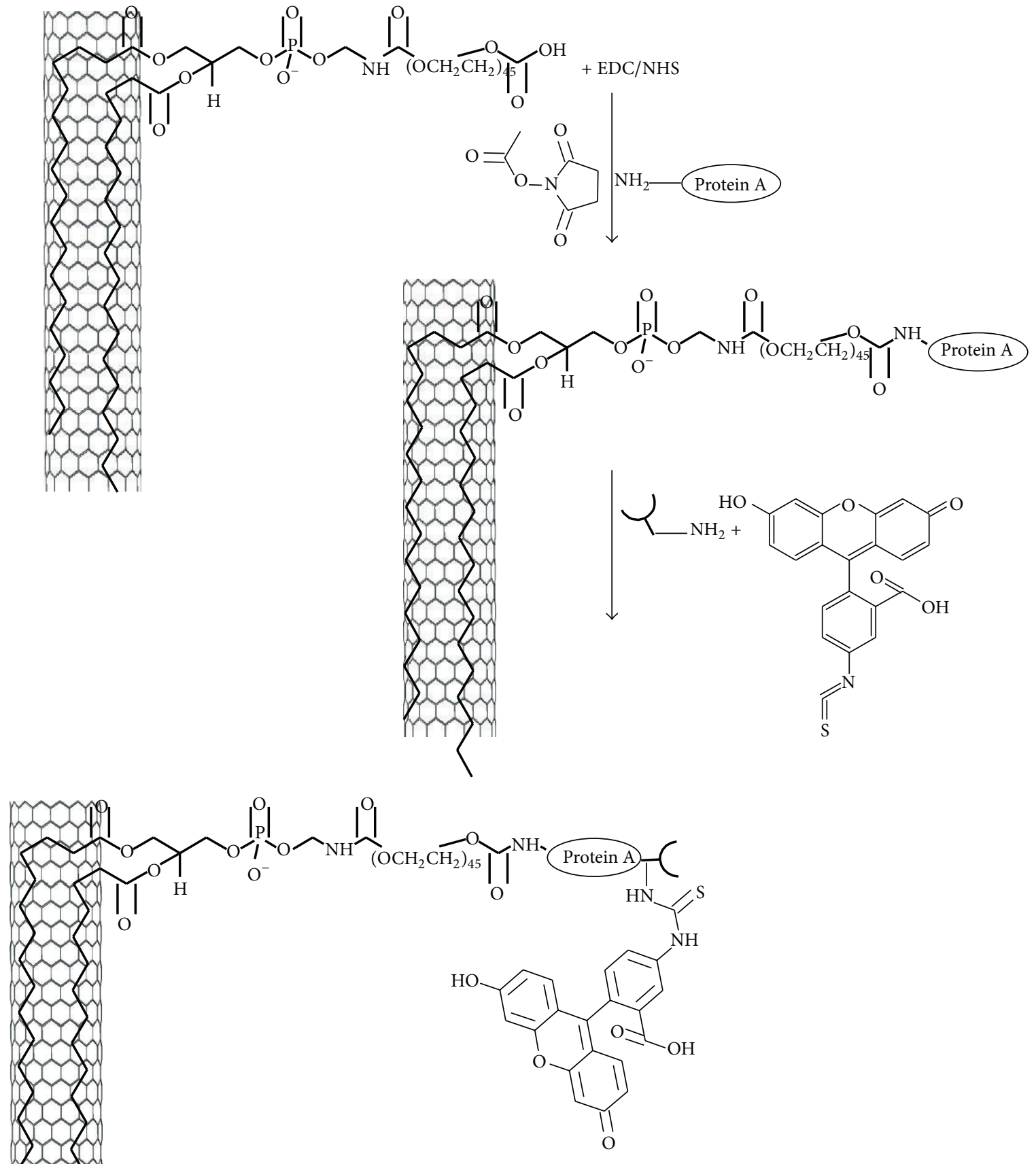

Scheme 2

any side effects. Nanoparticles had higher loading capacity of doxorubicin comparing with liposomes.

The bioconjugation of protein to nanocarrier by thioether bond was described by Gindy's group [98]. The carrier EG- $b$-PCL was modified by maleimide. The BSA was used as targeted ligand containing $\mathrm{SH}$ fragments. The process occurred in aqueous solution. The covalent bioconjugation between albumin and nanocarrier was confirmed by the analysis of the nanocarrier volume before and after the process. Higher concentration of protein in solution during the reaction caused increase in the number of BSA-nanoparticle bonds. The targeted nanoparticles modified by thioether bond between ligands, that is, aptamers and nanocarrier, were shown by Farokhzad et al. [99] and Xiao et al. [100]. 


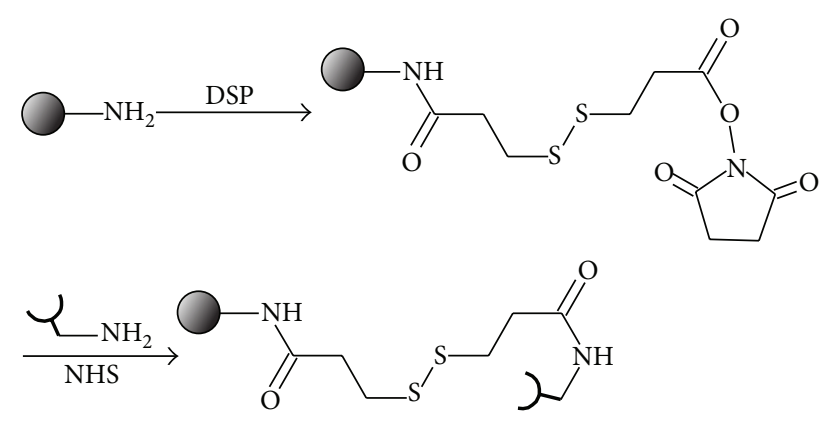

SCHEME 3

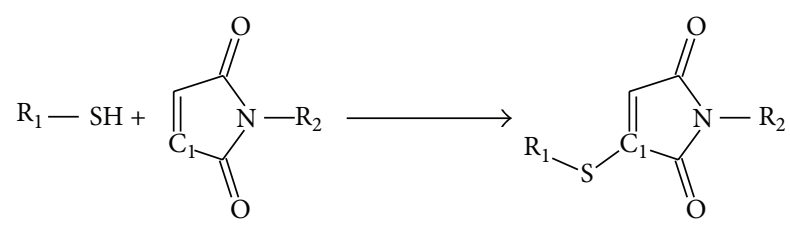

SCHEMe 4

The nanoparticle-aptamers system was successfully applied in anticancer therapy. The therapeutic effect was enhanced. Also, Lu et al. [101] successfully applied thioether bound between folic acid and functionalized carbon nanotubes. This new system was characterized in special properties to the application in targeted delivery of DOX against cancer treatment.

Alternative way is the coupling of thiol groups of the nanocarrier with maleimide fragments from the ligand. The thiol groups could be activated via amines or carboxylic acid groups (Scheme 6).

The amide and thioether are the main bond types used to form the ligand-nanocarrier systems. Other connection types are only the derivatives [12].

4.3. Disulfide Group. The disulfide bond is formed by the conjugation of two thiol groups (Scheme 7). The first group originates from a nanocarrier while the other from ligand.

The reduction of disulfide functionalities [102] or the application of suitable agents as SATA or SPDP [103] can form thiol groups of a ligand. In order to form the thiol groups on nanocarrier surface, one should modify it with PDP-PE, PDPSA, and PDP-PEG-DSPE, where PDP fragment is a source of thiol groups [12].

In [104] the formation of disulfide bond between liposomes and monoclonal antibody anti-My9 was described. The carrier surface was decorated with PDP-SA. The ligand was modified with SPDP and kept its own immunoreactive level after the modification. The obtained system in this way acts strongly against human HL-60 promyelocytic leukemia cells [104]. The formation of disulfide groups between targeted ligands (nanobodies) and micelles was shown by Talelli et al. [105].

4.4. Acetyl-Hydrazone Group. The conjugation of hydrazide groups to the nanocarrier surface occurs with aldehyde groups of the ligands (Scheme 8) [77,78]. As generally ligands do not possess the aldehyde structure, the latter must be formed via oxidation of hydroxyl groups. The oxidative agents are usually sodium periodate [106] and galactose oxidase [107]. The major advantage of this method is the rigorous control of the ligand modification [108]. However, the yield of the process in this technique is very poor [93].

Harding et al. [109] described the linking of antibodies C225 with liposome through acetyl-hydrazone group formation. The in vivo study showed that the activity of antibody in produced immunoliposome was fully kept. The system was characterized by long time of distribution in blood and high immunological level. Using this methodology, one can easily control immunoliposome-structure synthesis.

4.5. Polycyclic Group. The Diels-Alder reaction (Scheme 9) is the cycloaddition reaction between a diene and a dienophile. As a result, bicyclic compound is formed. The coupling of ligands and nanocarriers is beneficial because of high yield (close to 100\%) and easy synthesis under mild conditions [77].

The system of ligand-nanocarrier formed in the DA reaction causes specific bonds creation between ligand and cancer cells [12]. Shi et al. [110] used the DA reaction to conjugate antibody anti-HER2 with the polymeric nanocarrier (Scheme 10). The carrier was synthesized via a soft templating method. The furan group on the external surface of carrier was the diene while maleimide group of antibody was the dienophile. Authors demonstrated the ability of this technique in creation of bioactive immunonanoparticles and concluded that the versatility of the nanoparticle system can be extended to create multiple functional delivery vehicles. Formed immunosystem was tested against breast cancer [111, $112]$.

4.6. The "Click Chemistry". The term "click chemistry" (CC) was developed by Kolb's group [113] and describes the coupling of small elements and heteroatoms in the form of R-X-R. Reactions belonging to "click chemistry" group are characterized by high efficiency, stereospecificity, and harmless side products. These processes need (i) mild conditions, (ii) readily available reagents, and (iii) easily removed solvent, for example, water [77].

Formed product should be stable under physiological conditions and should be easily isolated. The main method of the purification of final product is crystallization or distillation [113]. Within the CC, one can find four major types $[113,114]$ :

(i) Cycloadditions, for example, Huisgen catalytic cycloaddition (Scheme 11);

(ii) nucleophilic substitution chemistry, for example, ring opening of heterocyclic electrophiles (Scheme 12);

(iii) carbonyl chemistry of the "nonaldol" type, for example, formation of ureas, thioureas, and hydrazones (Schemes 13-14); 


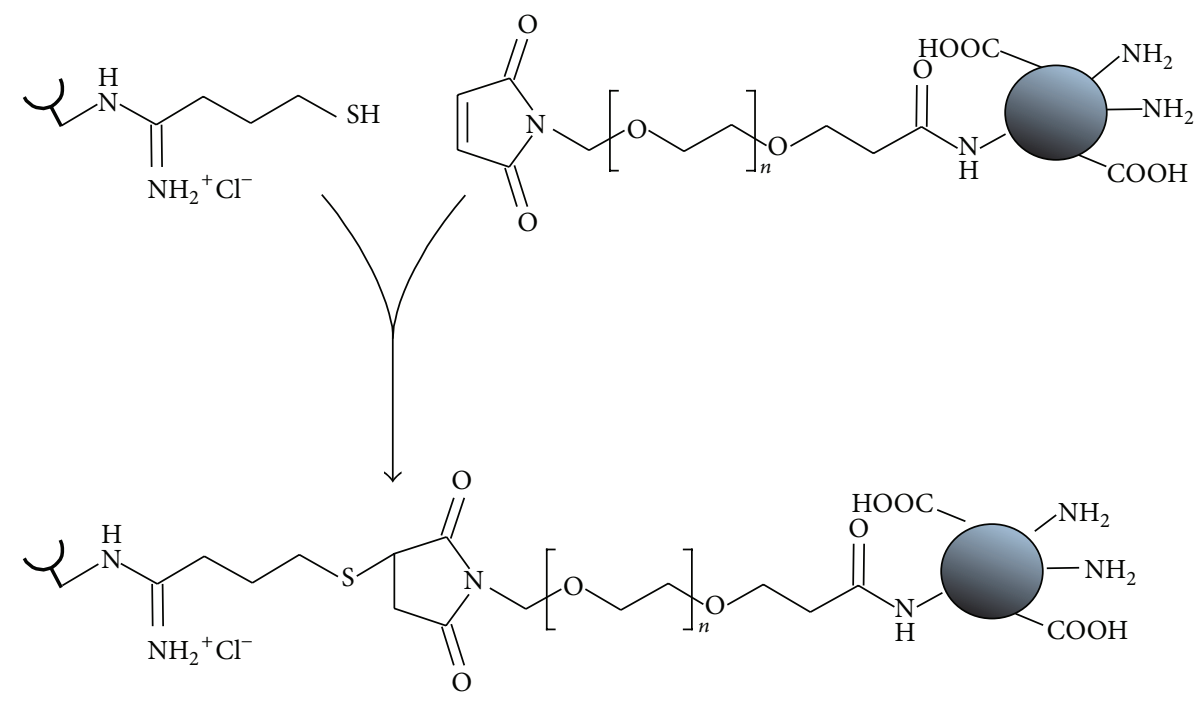

SCHEMe 5

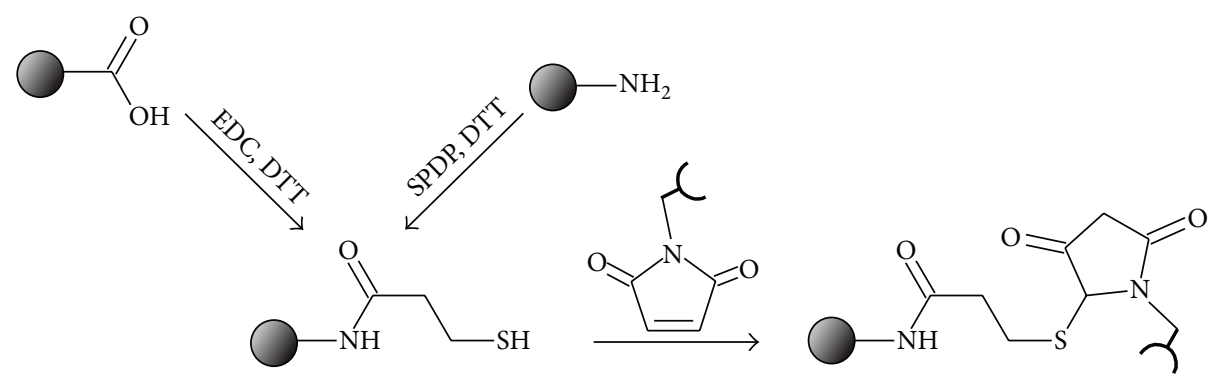

SCHEME 6

$\mathrm{R}_{1} \longrightarrow \mathrm{SH}+\mathrm{HS} \longrightarrow \mathrm{R}_{2} \longrightarrow \mathrm{R}_{1} \longrightarrow \mathrm{S} \longrightarrow \mathrm{S} \longrightarrow \mathrm{R}_{2}$ SCHEME 7
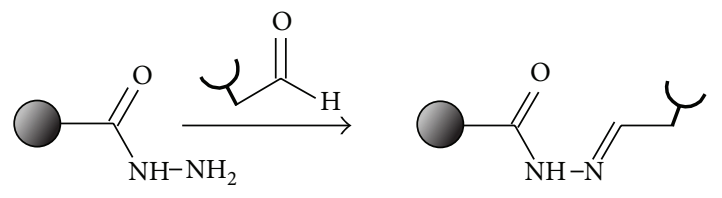

SCHEMe 8

(iv) additions to carbon-carbon multiple bonds, for example, epoxidation and dihydroxylation (Schemes 1516).

The major type of "click chemistry" reactions is the Huisgen cycloaddition. This process creates 1,2,3-triazole by the 1,3-dipolar cycloaddition between azides and terminal alkynes in the presence of the catalyst, $\mathrm{Cu}$ (I) $[12,77,115]$. The most probable mechanism of the reaction is shown in Scheme 17 [114].

Huisgen's cycloaddition requires reagents tolerating aqueous solution with broad range of $\mathrm{pH}$ level and biological molecules [114]. The effective ligand-liposome conjugation

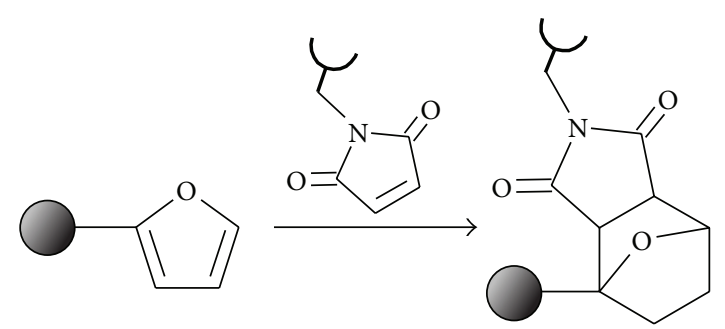

SCHEMe 9 based on Huisgen's cycloaddition was described by Hassane et al. [116]. The ligand was $\alpha$-D-mannose derivative with azide group. The liposome possessed groups with triple bonds. Reported studies confirmed the essential role of addition of $\mathrm{Cu}(\mathrm{I})$ in order to increase efficiency. The synthesized product was almost perfect selective immunosystem [116].

The De et al. [117] results confirmed the possibility of "click chemistry" application in targeted anticancer therapy in order to conjugate the folic acid to the micellar nanocarrier. Also, Lu et al. [118] decorated nanoparticles by selective ligands using Huisgen's cycloaddition. The process was effective and almost 400 peptide molecules, modified coumarin, were attached to each nanoparticle. Fluorescence intensity of 


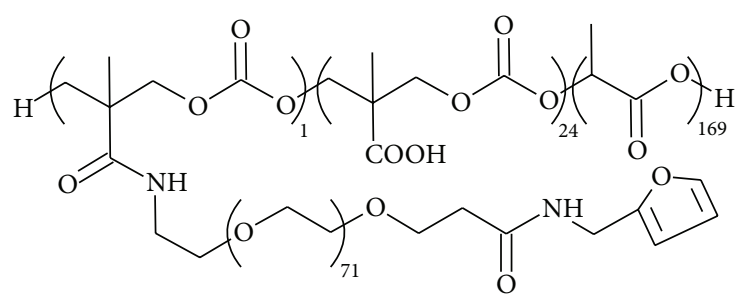

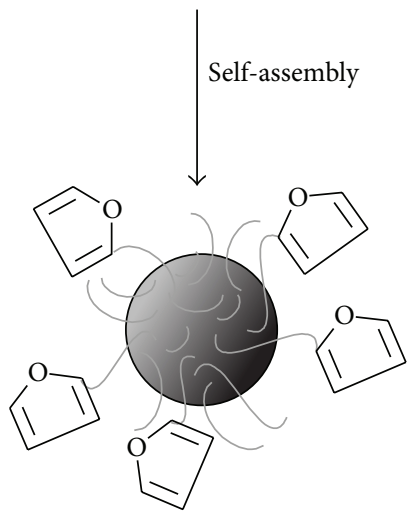

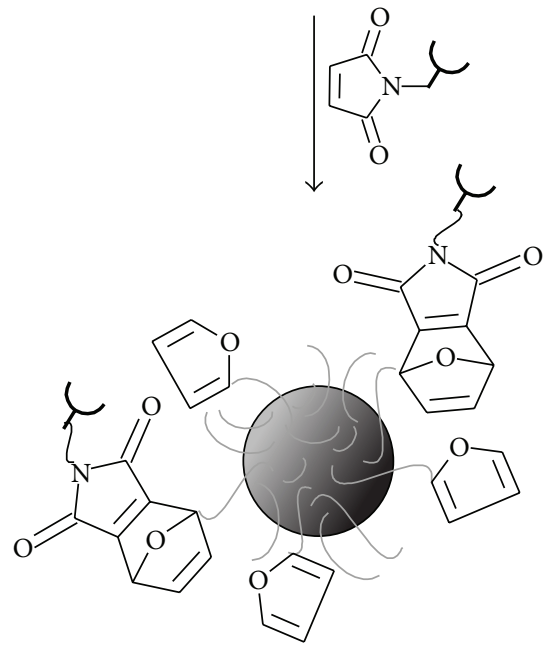

SCHeme 10

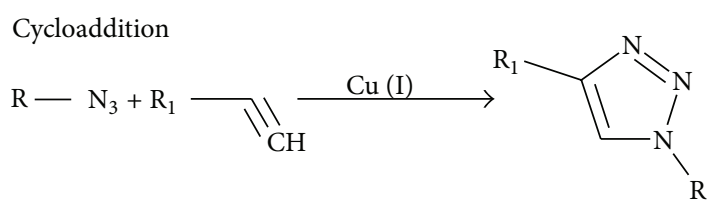

SCHEME 11

coumarin confirmed the efficiency of the reaction between peptide connecting alkyne group and nanoparticle with azide group [118]. Carbon nanotubes can also be modified by Huisgen's cycloaddition. Carbon nanotubes functionalized by Huisgen's cycloaddition were used in delivery of methotrexate to breast cancer cells [18].

We described here only the most popular covalent ligandnanocarrier bonds which are often used in practice. However, up to date, much more types of covalent bonds are known. The other, covalent link, carbamate bond, between targeted ligand and liposomes was shown by Sawant and Torchilin [119]. An alternative and effective functionalization method is the formation of amide bonds between azides and triphosphine known as Staudinger's reaction. Generally, it occurs at room temperature and in aqueous solution and does not require any catalyst. Zhang et al. [120] attached glycoliposome with lipid containing triphosphine fragments by Staudinger's reaction. This method is applicable to water soluble molecules.

Another type of covalent bond is the immobilization of primary amine with free aldehyde group of ligand by formation of Schiff's base [78]. This technique was used during the linking of the transferrin (TF) with poly(lactic acid) surrounded by cholesterol chains containing indomethacin molecules. The TF-nanocarrier was studied against glioma cell. Experiment confirmed the bioactivity of transferring [121].

The main covalent interactions and essential conditions of the processes are shown in Table 1.

\section{Physical and Chemical Bonding of Drug to Nanocarrier Surface}

Drugs applied in targeted anticancer therapy could be accumulated outside and/or inside of nanocarriers (Figure 4). The couplings can be nonspecific (e.g., adsorption) or covalent [122].

The entrapment of a drug to nanocarrier is possible during the synthesis of nanocarrier or after the carrier formation. The loading of chemotherapeutic agent depends on its solubility in the nanoparticle matrix, the mass of the molecule, the interaction of drug nanocarrier, and the type of functional groups present on a nanocarrier surface [123]. A nanocarrier should be chemically resistant and should possess high purity. These properties are essential to control delivery of drug [124].

Nanocarriers are often modified before the entrapment of drugs. The most popular method of functionalization employs polymers. The medical practice uses both natural and synthetic polymers, for example, polyethylene glycol, poly(lactic acid), N-(2-hydroxypropyl)methacrylamide copolymer, poly(L-glutamic acid), and poly(D,L-lactic-coglycolic acid) [124]. The bioconjugation of drug with polymer leads to the extension of chemotherapeutic circulation and controlled drug release in targeted place [125-129]. PEG has high solubility in water. Moreover, it is a good "candidate" for application in medicine because of its biochemical properties: biodegradation, minimal toxicity, and controlled mechanical properties $[125,130]$. Generally, it is important to apply the polymeric phase because it improves the drug accumulation in targeted place and generates the barrier between nanocarrier and cancer cell $[126,129]$.

5.1. Drug on the Internal Surfaces Nanocarriers. The internal walls of a nanocarrier (e.g., of CNT) in contrast to the external walls reveal high interaction energy with adsorbed molecules. The entrapped drug molecules inside the nanocarrier are 
TABLE 1: Types of covalent ligand-carrier bindings.

\begin{tabular}{|c|c|c|c|c|}
\hline Name & $\begin{array}{c}\text { Functional group of } \\
\text { nanocarrier }\end{array}$ & $\begin{array}{l}\text { Functional group of } \\
\text { ligand }\end{array}$ & Conditions of process & Literature \\
\hline Amide group & Carboxylic & Primary amine & $\begin{array}{c}\text { EDC, NHS, pH 7.5-8.5, } \\
2-24 \mathrm{~h}, 4^{\circ} \mathrm{C} \text { lub RT }\end{array}$ & {$[98,102-108]$} \\
\hline Thioether group & Maleimide & Thiol & $\mathrm{pH}>7,4-24 \mathrm{~h}, \mathrm{RT}$ & {$[102,111-117]$} \\
\hline Acetyl-hydrazone group & Hydrazide & Hydroxylic & $24 \mathrm{~h}, 5-6^{\circ} \mathrm{C}$ & {$[124]$} \\
\hline Disulfide group & Thiol & Thiol & $\mathrm{pH} 8.0,2-24 \mathrm{~h}, 4^{\circ} \mathrm{C}$ or RT & {$[120]$} \\
\hline Diels-Alder & Furan & Maleimide & $\mathrm{pH} 5.5,2-6 \mathrm{~h}, 37^{\circ} \mathrm{C}$ & {$[125,126]$} \\
\hline “Click chemistry” (HDC) & Azide & Alkyne & $\mathrm{Cu}(\mathrm{I}), \mathrm{RT}, 2-3 \mathrm{~h}$ & {$[131-134]$} \\
\hline Staudinger & Azide & Triphosphine & PBS, pH 7.4, 6 h, RT & {$[135]$} \\
\hline Schiff's base & Primary amine & Aldehyde & $\mathrm{pH} 9.2, \mathrm{RT}$ & [138] \\
\hline
\end{tabular}

Nucleophilic substitution chemistry

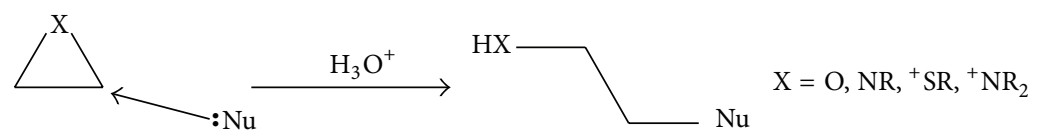

SCHEME 12

"Nonaldol" carbonyl chemistry

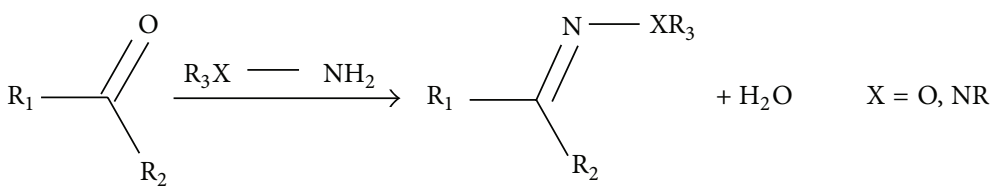

SCHEME 13

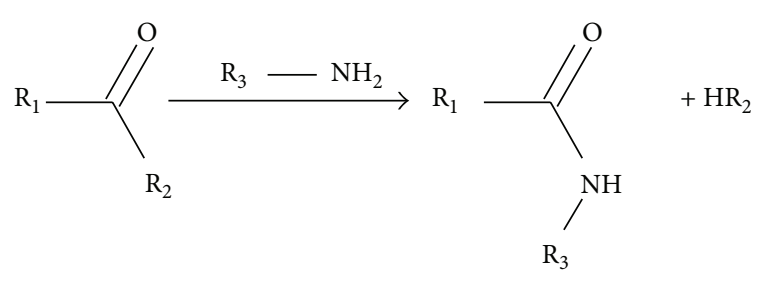

Scheme 14

isolated from external environment and they are protected against early activation and degradation process. Thus, the interaction of chemotherapeutic with healthy tissue is impossible $[131,132]$.

Drug molecules can be entrapped by nanoextraction and/or nanocondensation process (Figure 5). Nanoextraction process occurs in liquid phase and at the room temperature. The idea is based on the selection of solvent adequate to the guest molecule. Entrapped drugs should have poor affinity to the solvent and high affinity to the nanocarrier. Weak solubility of guest molecules in the solvent enables formation of the suspension in liquid phase and the gradual diffusion inside the nanocarrier [133].

Nanocondensation, similarly as nanoextraction process, is performed in liquid phase also at the room temperature.

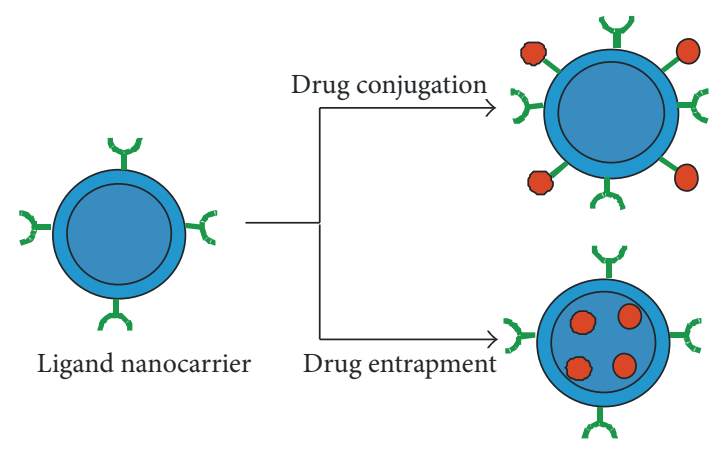

FIgURE 4: The drug conjugation to surface of a carrier and an entrapment process (the figure is based on [122]).

This process is relatively easy and quick. Guest molecules should demonstrate high affinity to the solvent and to the nanocarrier. Generally, nanocondensation process is based on the adsorption of solvent molecules on the external and internal walls of nanocarriers. Guest molecules migrate through the thin layer of a solvent and are adsorbed in the most active centers via the van der Waals interactions [133].

Nanoextraction process was utilized by Ren and Pastorin [19]. Authors loaded the drug, hexamethylmelamine, inside 
Carbon multiple bond addition

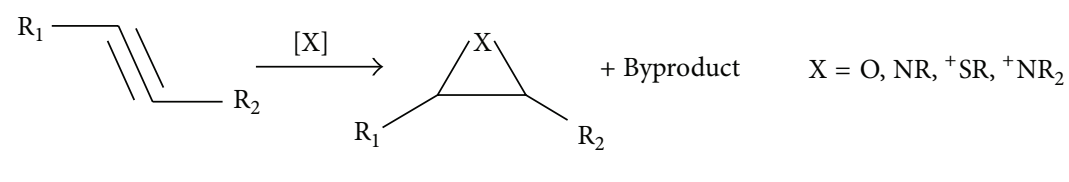

SCHEME 15

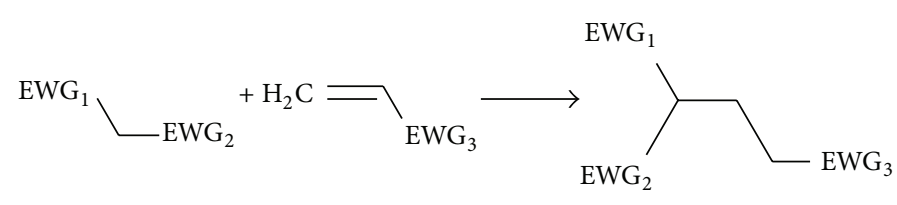

Scheme 16
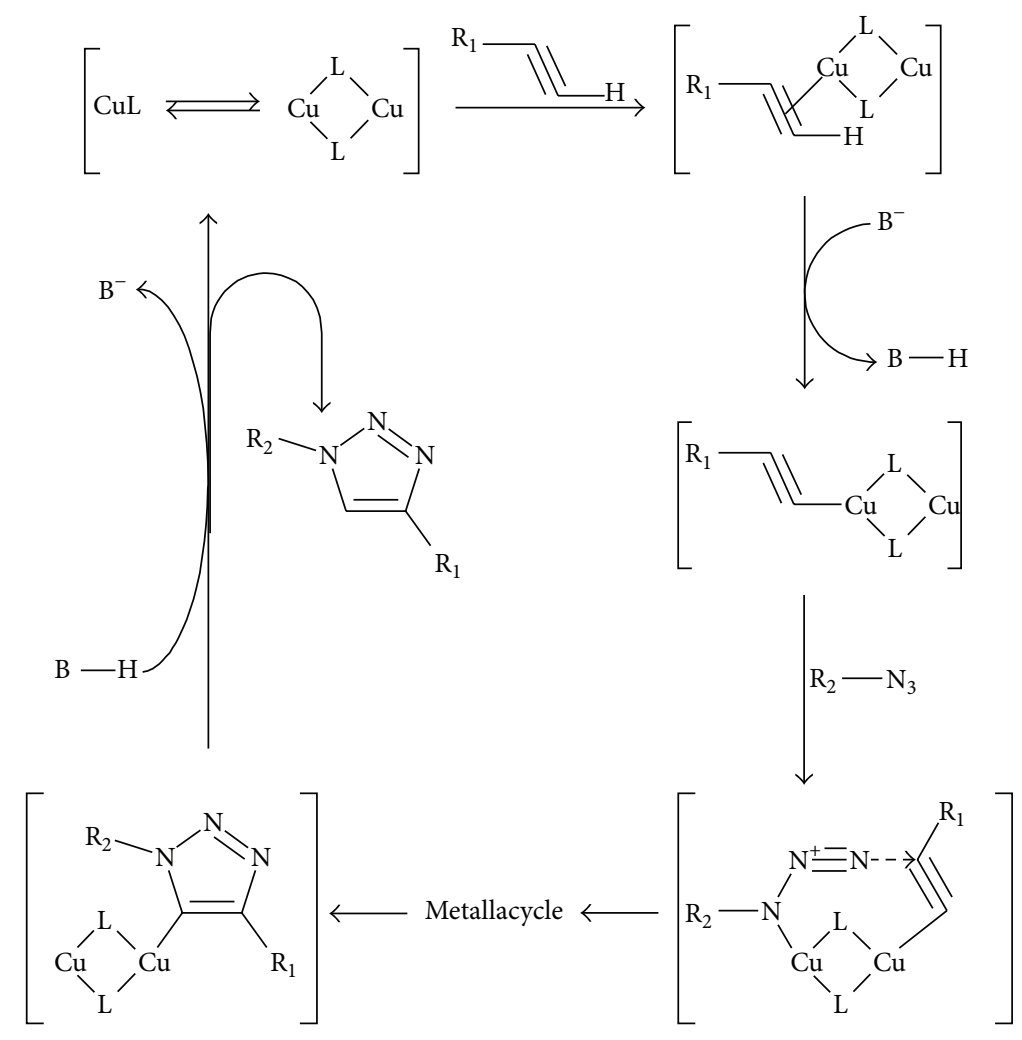

SCHEMe 17

CNTs. C60-fullerenes were used as caps of open CNTs ends. In the first step, CNTs were treated by a mixture of acids $\left(\mathrm{HNO}_{3}, \mathrm{H}_{2} \mathrm{SO}_{4}\right)$. During this process, CNTs were opened and functional groups (mainly carboxylic) were generated. Carboxylic fragments created the barrier for other molecules compared to the drug ones. Authors proved that suitably functionalized CNTs are good candidates for drug nanocarriers. The stable structures of CNTs and C60 protected drug molecules from circulation in blood before they were released at specific places [19].

Studies concerning the cisplatin loaded inside SWCNTs were reported by Tripisciano et al. [134]. The system of SWCNTs-cisplatin was used against colon cancer. Authors utilized a mixture of $\mathrm{HNO}_{3}$ and $\mathrm{H}_{2} \mathrm{SO}_{4}$ acids in order to open SWCNTs. The drug was loaded inside SWCNTs by nanocondensation process. Carbon material was dispersed in dimethylformamide (DMF) containing cisplatin (DDP). Carbon nanotubes limited the drug precipitation to aqueous solution and its degradation. Analysis of FTIR, Raman spectroscopy, HR-TEM, and EDX results confirmed the presence of cisplatin inside SWCNTs structure. Authors observed the proportional correlation between the anticancer properties and the amount of SWCNTs-DDP. The new DDS caused similar effect on the cancer cells as free drug. However, the novel system minimized the presence of side effects. Another work of Tripisciano et al. [135] reported the nanocondensation 
TABLE 2: Effectiveness of DDP delivery by SWCNTs [134] and MWCNTs [135].

\begin{tabular}{lcc}
\hline & SWCNTs & MWCNTs \\
\hline The amount of DDP inside CNTs & $21 \mu \mathrm{g} / 100 \mu \mathrm{g}$ of CNTs & $13.6 \mu \mathrm{g} / 100 \mu \mathrm{g}$ of CNTs \\
The amount of released DDP & $68 \%$ & $95 \%$ \\
The rate of DDP release & Slower & Faster \\
The maximum release of DDP & $72 \mathrm{~h}$ & $48 \mathrm{~h}$ \\
\hline
\end{tabular}

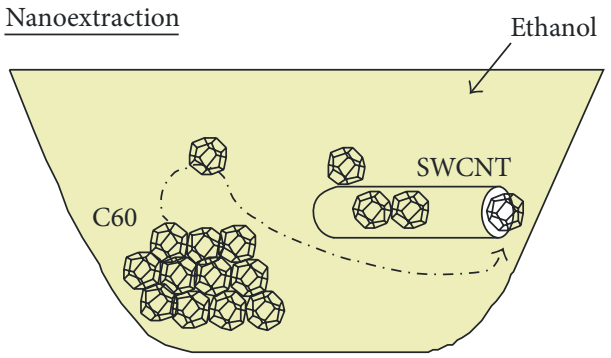

Nanocondensation
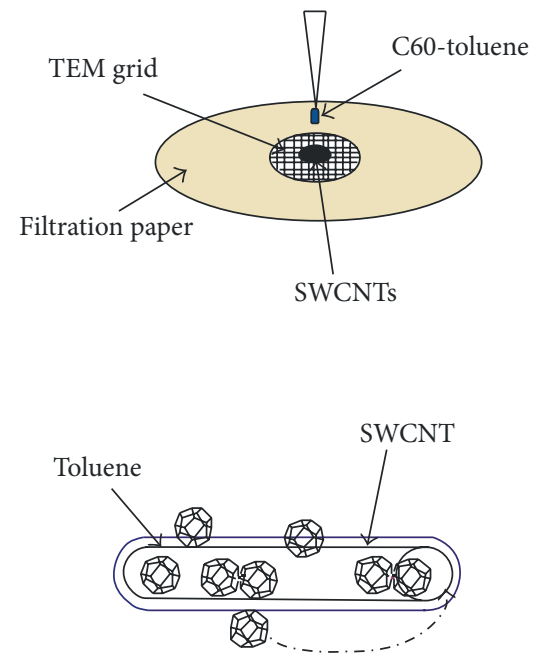
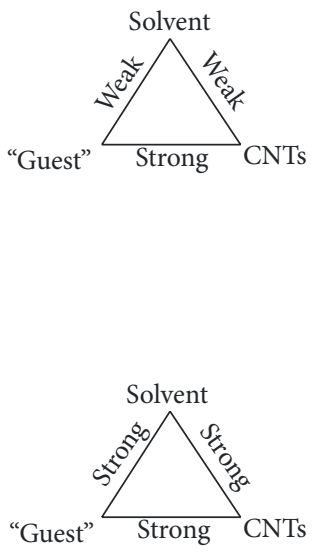

FIGURE 5: Scheme of the nanoextraction and nanocondensation processes (the figure is based on [133]).

process used to the loading of cisplatin inside the MWCNTs. The method of entrapment process was similar to SWCNTs. Table 2 summarizes the results for SWCNTs and MWCNTs.

It is interesting that almost all drug molecules were released from MWCNTs. This confirms the fact that carbon nanotubes can be successfully used as drug containers and therefore they could serve as DDS. Moreover, according to the authors, inside DDP-MWCNT systems the specific interaction did not occur, in contrast to DDP-SWCNTs. Those interactions are probably responsible for slower and smaller percentage release of DDP from SWCNTs. Likewise, Li et al. [136] and Sui et al. [137] described the encapsulated DDP inside carbon nanotubes. Authors confirmed the improved efficiency of anticancer therapy.

Another interesting approach is the entrapment of DDP inside the nanoparticle covered with copolymer. Gryparis et al. [138] used this container against colon cancer. The drug kept its activity after loading inside the nanocarrier. It was delivered selectively to cancer cell. The therapeutic effect was better comparing with the traditional chemotherapy. The DDP-nanoparticle system was safe for human organism. Cisplatin can be also adsorbed within PMs [139, 140] or liposomes [141, 142] or carbon nanohorns [143, 144]. The in vivo and in vitro studies were aimed against lymphoma (J6456) [141], colon (C26) [141, 142], lunge [142-144], and stomach (MKN 45) [140] cancer cell. Analysis confirmed that the DDP internalization inside nanocarriers improved the drug activity and minimized side effects [139-142].

Hampel et al. [145] used the entrapment process of carboplatin inside carbon nanotubes. They were opened and next the drug was placed inside the structure using wet impregnation method. Authors confirmed the presence of carboplatin inside the structure which protects the drug against the environment. Carboplatin inhibited the growth of blood cancer 


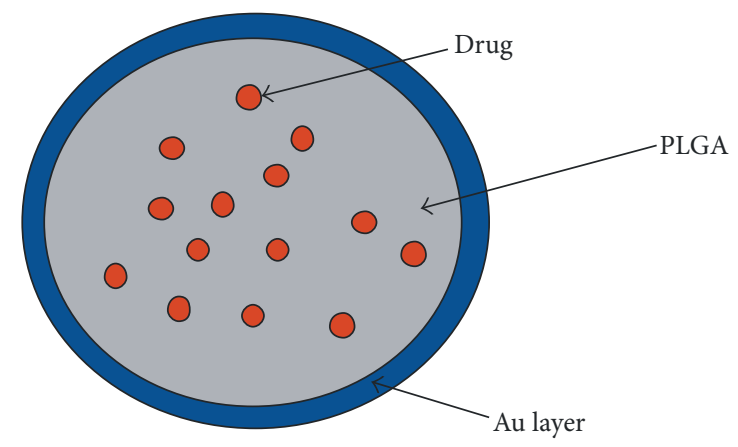

Figure 6: Schematic diagram of DOX-PLGA-Au (the is figure based on [149]).

cells. The results confirm efficiency of carbon nanotubes as chemotherapeutic nanocarriers [145]. In another interesting work [146], an encapsulated Pt(IV) prodrug inside polymeric nanoparticle was described. Additionally, the targeted ligands were conjugated to the surface of nanoparticle. This DDS inhibited growth of breast as well as prostate cancer cells. This fact was connected with the protection of prodrug structure by nanoparticles. Moreover, the biodistribution of novel system was better than that in the case for cisplatin. Mitra et al. [147] reported the DDS based on doxorubicin inside nanoparticle (diameter ca. $100 \mathrm{~nm}$ ). Drug was linked with additional remedy, dextran. The conjugation limited side effects of doxorubicin. The nanoparticle-DOX system improved therapeutic effect of drug. Authors observed the reduction of tumor volume after 4 weeks. Doxorubicin time circulation in blood and amount inside cancer cell were better in comparison to the traditional chemotherapy.

Lince et al. [148] and Park et al. [149] described process of doxorubicin entrapment inside nanoparticle. Lince et al. [148] showed nanoparticle based on biodegradable and biocompatible copolymer. The polymeric nanocarrier improved activity of drug which was placed inside and limited its potential side effects. Thus, the comfort level and quality of patient's life increased. Park et al. [149] as well as Lince et al. [148] described the encapsulation of doxorubicin inside polymeric nanoparticle. The polymer nanocarrier was covered with the extra gold layer (Figure 6). The DOX-PLGA-Au system combined traditional chemotherapy and phototherapy. The efficiency was examined against cervical carcinoma. The novel therapy reveals high therapeutic efficiency and short time of treatment. The DOX-PLGA-Au system delivered selectively drug to cancer cell and thus caused rise of temperature, which destroys cancer tissues. The warmth was generated as a result of absorption of NIR radiation by gold layer.

Doxorubicin in targeted anticancer therapy was used successfully within liposomes by Matsumura et al. [150] or Gabizon [151] and within micelles by Kataoka et al. [152], Perche et al. [153], Ebrahim Attia et al. [154], or Cambón et al. [155]. Paclitaxel similarly as cisplatin or doxorubicin can be adsorbed inside the nanocarrier. Koziara et al. [156] used this drug inside nanoparticle in vitro against brain cancer cells U-118 and HCT-15. Authors proved the rise in cytotoxic

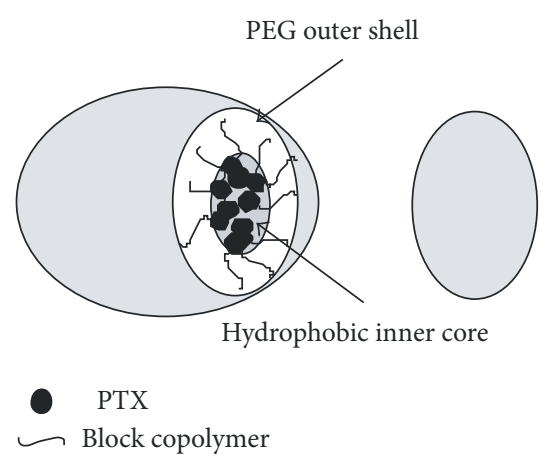

FIGURE 7: Schematic structure of NK105 (the is figure based on $[160])$.

activity of drug and the limitation of the transmembrane pump action. Thus, the uptake of a drug by cancer cell was facilitated. The new system penetrated through bloodbrain barrier. The stability of PTX was maintained during the entrapment process. Ruan and Feng [157] proved that the release from nanoparticle is easy when the nanoparticle is covered with copolymer PLA-PEG-PLA. Probably this effect occurs because hydrophilic fragments of PEG inside hydrophobic fragments of PLA increase the porosity [157]. Fonseca et al. [158] and Chan et al. [159] also described the immobilization of paclitaxel inside polymer nanoparticles. Authors proved the efficiency of this method [158]. Hamaguchi et al. [160] proposed the new form of PTX-NK105, namely, the drug inside micelle (Figure 7).

The delivery of NK105 to organism runs without Cremophor EL and ethanol in contrast to the traditional anticancer therapy. The NK105 shows lower toxicity against nervous system and better activity [160]. Similarly, Kim et al. [161] and Wang et al. [162] located paclitaxel within polymer micelle. They confirmed the efficiency of novel form.

5.2. Drug on the External Surfaces of Nanocarriers. To construct the delivery system with a drug on external surface of a nanocarrier, the chemical or physical conjugation is applied. This method is based on chemical properties and high surface area of a nanocarrier [132]. Drug molecule is linked with functional groups of nanocarrier or polymer covering nanocarrier by covalent bonds forming ester, amide, or acetyl-hydrazone groups. This type of coupling has usually low stability [163] and shows pH sensibility [164]. Drugs with plane or aromatic structure can be adsorbed on the surface of nanocarriers by $\pi-\pi$ interactions [163].

5.2.1. Ester Group. The ester bond formation between, for example, doxorubicin and nanocarrier, is possible as a result of

(i) the bioconjugation of drug's primary hydroxylic group $\left(-\mathrm{C}=\mathrm{OCH}_{2} \mathrm{OH}\right)$ with polymer's carboxylic group, for example, PLGA surrounding nanocarrier (Scheme 18);

(ii) the bioconjugation of drug's primary amine group with polymer's hydroxylic group (Scheme 19). 


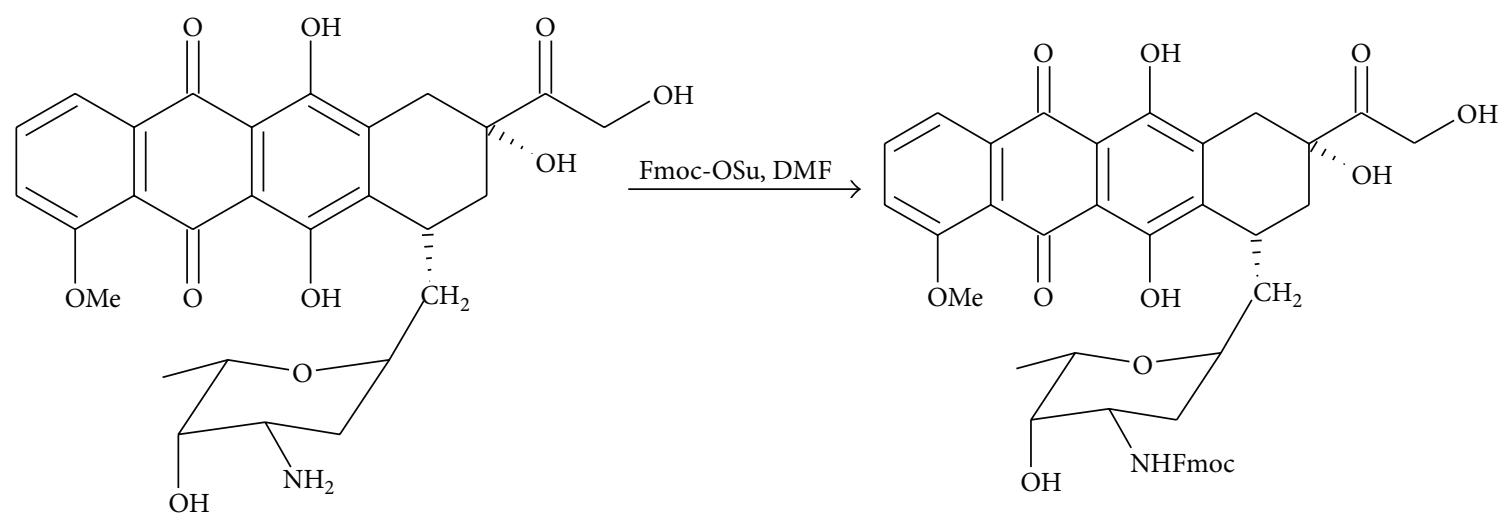

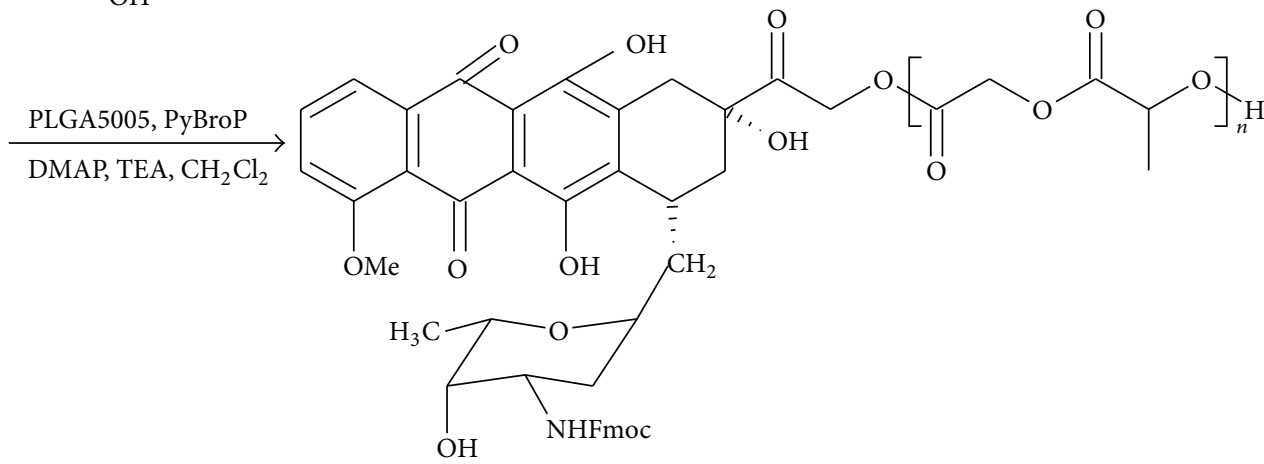<smiles>COC(=O)COC(=O)C(C)OCC(=O)C1(O)Cc2c(O)c3c(c(O)c2[C@@H](CC2CC(N)C(O)C(C)O2)C1)C(=O)c1c(OC)cccc1C3=O</smiles>

SCHEME 18

Yoo et al. [127, 128] described the formation of ester bond between DOX and nanocarrier covered with PLGA. The conjugation takes place between hydroxylic and carboxylic groups [127] and between amine and hydroxylic groups [128]. The novel systems were in vivo and in vitro tested against cancer cell line HepG2. For both of the cases, drug delivered on the external surface was better absorbed by cancer cell than the drug placed inside nanocarrier. Authors showed that the release of drug from nanocarrier is correlated with the molecular mass of polymer placed on the surface of nanocarrier [127].

Another anticancer drug as, for example, paclitaxel, can be conjugated to nanocarrier by ester bond. Li et al. [165] showed the bioconjugation of paclitaxel with polymeric nanocarrier covered by poly(L-glutamic acid) (PG). The therapeutic activity of drug against $\mathrm{OCa}-1$ cancer cells was higher than during traditional chemotherapy. Authors proved that the PTX-PG conjugation has lower toxicity than free PTX. The novel system was better bonded to cancer cells and its circulation time was longer. It was confirmed that labile ester bond formation between polymeric nanocarrier and chemotherapeutic agent is useful method for the designing of targeted DDS. Milas et al. [166] used the same idea to link paclitaxel with polymeric nanocarrier and confirmed the efficiency of the method.

5.2.2. Acetyl-Hydrazone Group. The hydrazone bond can be formed via the conjugation of carbonyl group with hydrazide one. This nanocarrier-drug linking has high cytotoxicity level against selected cancer cell lines [167]. Doxorubicin possesses the carbonyl group which can combine with hydrazide group on the surface of nanocarrier covering polymer [168]. The most popular polymers described in the literature are poly(allyl glycidyl ether) [169] (Scheme 20), 
<smiles></smiles><smiles>CC(OC(=O)COC(=O)C(C)OC(=O)COC(=O)Oc1ccc([N+](=O)[O-])cc1)C(=O)O</smiles>

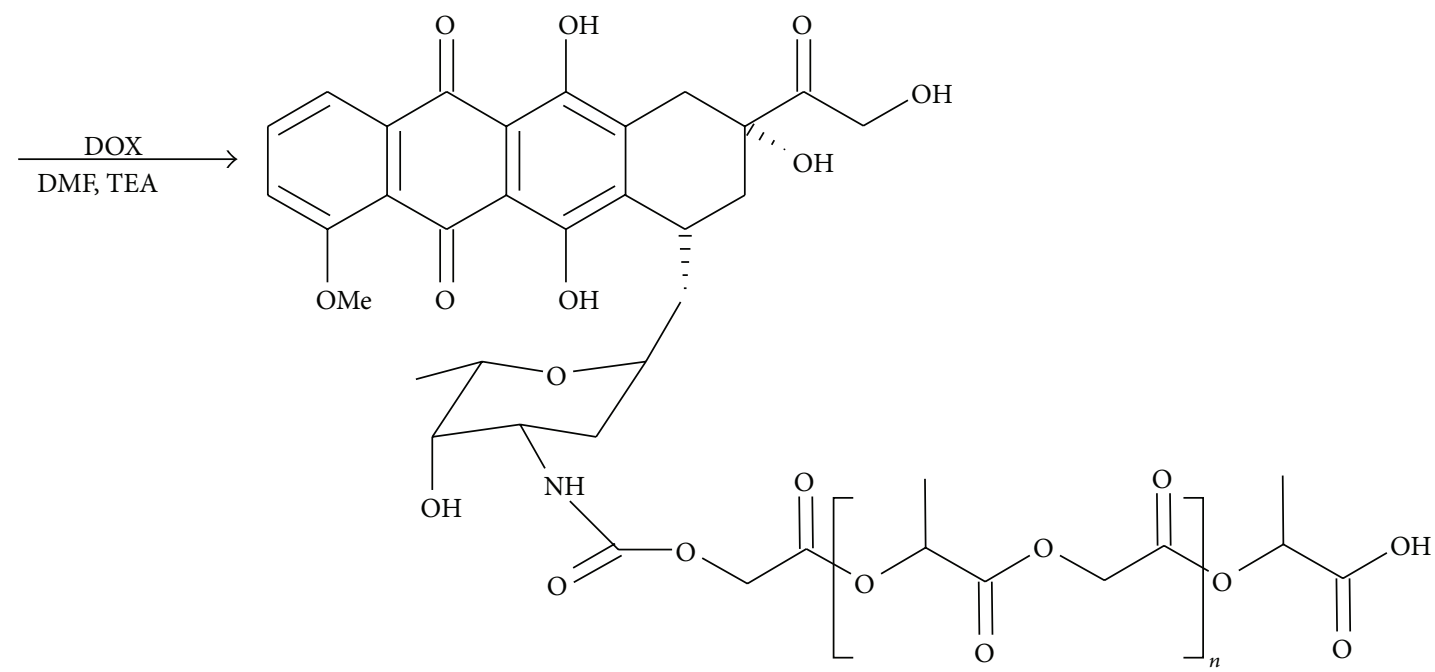

SCHEME 19

N-(2-hydroxypropyl)methacrylamide copolymer [167], and poly(ethylene oxide)-block-poly(allyl glycidyl ether) [168].

Vetvicka et al. [169] proposed micellar arrangement based on amphiphilic, diblock copolymer, and doxorubicin. This system shows 20-time lower toxicity and longer time of circulation than the free drug. The therapeutic activity against lymphoma EL-4 T was promising, and 75\% of mice population was cured completely and showed specific resistance against new cancer cells [169].

Hrubý et al. [168] as well as Liu et al. [170] described the formation of acetyl-hydrazone group between doxorubicin and nanocarrier covering poly(ethylene oxide)-blockpoly(allyl glycidyl ether) [168] and poly(ethyleneimine)polyethylene glycol [170], respectively. Drug release was $\mathrm{pH}$ sensitive: it was faster in $\mathrm{pH}$ similar to that of endosomes $(\mathrm{pH}$ $5,0)$ than in $\mathrm{pH}$ of blood plasma $(\mathrm{pH} 7,4)$. Probably, this effect is related to the shift of equilibrium between free and bonded molecules of doxorubicin to the direction of drug release in lower $\mathrm{pH}$ [168]. Yoo et al. [171] showed the hydrazone bond between doxorubicin and polymeric micelles. Drug was linked to the terminal hydrazide groups on the nanocarrier, poly(lactic acid) and methoxy-polyethylene glycol.
Doxorubicin was delivered quickly to the cancer cell and possessed high cytotoxicity [171].

Platinum drugs structural properties allow the formation of the hydrazone bond between drug and nanocarrier. Aryal et al. [172] described the bioconjugation of copolymer PEGPLA with the hydrazide moieties PEG-PLA-NH-NH $\mathrm{N}_{2}$ with levulinic acid modified with $\mathrm{Pt}(\mathrm{IV})$ cisplatin analogue. The amount of the associated drug molecules was controlled. Polymeric nanoparticles increased the cytotoxicity properties against ovarian cancer cells. The drug loss during nanocarrier circulation in the blood was low in natural $\mathrm{pH}$ [172].

The hydrazone bond is used for the bioconjugation of paclitaxel with dendrimeric nanocarrier. It was claimed that also polyamidoamine dendrimers (PAMAM) could be treated as nanocarrier of chemotherapeutic agents. The core is built up with alkyl-diamine with tertiary amine branches. Amine groups allow the easy functionalization of dendrimer with the drug. The modification of the nanocarrier surface by paclitaxel was used against ovarian cancer cells. The targeted ligand (protein LXW7) was also attached to the nanocarrier. The method was effective and eliminated cancer cells. The drug-nanocarrier bond was broken at low $\mathrm{pH}$ 


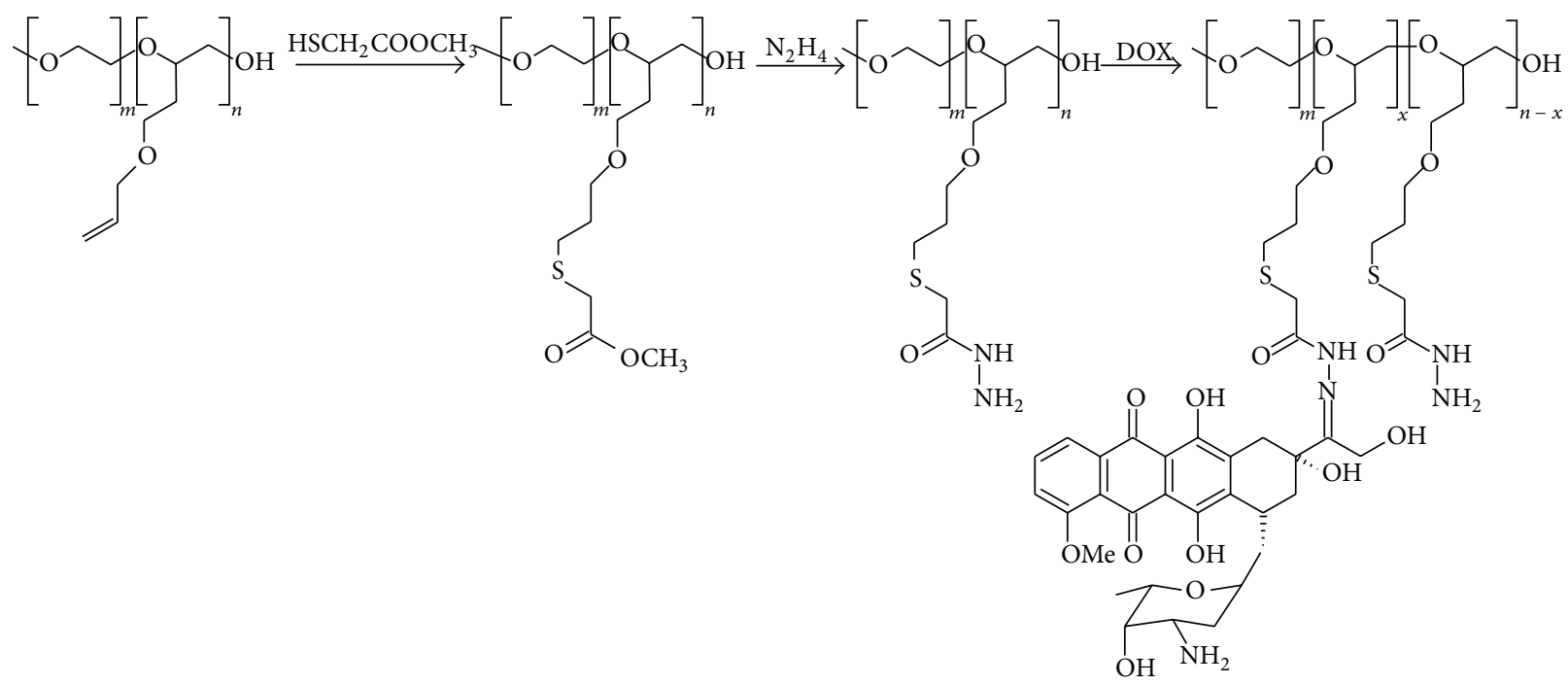

SCHEME 20

level and the drug was released [173]. Rodrigues et al. [174] demonstrated the bioconjugation of paclitaxel with polymeric nanocarrier, polyethylene glycol. The reaction required the preliminary modification of chemotherapeutic agent. The maleimide derivatives of paclitaxel were formed by bonding the maleimide fragments with drug molecule. At the first step, the synthesis of drug ester derivative in position C-2' $\mathrm{OH}$ by using 4-acetylbenzoic acid occurred. The intermediate product is a donor of carboxylic group which was essential to the attachment of maleimide derivative containing hydrazide fragment. Authors proved the efficiency of the method. The structure of drug was protected and its activity was improved via in vitro and in vivo studies.

5.2.3. Amide Group. Amide bond is formed during the reaction of carboxylic group with a nucleophile containing the primary amine. Feazell et al. [175] described the formation of amide bonds between platinum compound and SWCNTs. Functionalized SWCNTs were covered by phospholipid containing amine groups. The compound $c, c, t$ $\left[\mathrm{Pt}\left(\mathrm{NH}_{3}\right)_{2} \mathrm{Cl}_{2}(\mathrm{OEt})\left(\mathrm{O}_{2} \mathrm{CCH}_{2} \mathrm{CH}_{2} \mathrm{CO}_{2} \mathrm{H}\right)\right]$ as prodrug was bonded to the modified carbon nanotubes. The novel system was taken up effectively by endosomes, where $\mathrm{pH}$ level is low, and as a consequence cisplatin (DDP) which was released as active compound. This method enhances weak circulation and distribution of drug in blood and minimizes the DDP toxicity against healthy cells [175].

Liu et al. [48] described the conjugations between paclitaxel and SWCNTs by the amide bond. The structure of SWNCTs was functionalized by polyethylene glycol with amine fragment (PEG-NH$H_{2}$ ). The carboxylic group was introduced to the drug structure in $\mathrm{C}-2^{\prime}-\mathrm{OH}$ position. Drug showed better solubility than clinical form of paclitaxel, Taxol. Additionally, the SWCNTs-PTX system showed longer time distribution in the blood, the 10-time larger uptake by cancer cells, and stronger therapeutic effect in comparison to Taxol. The new form of paclitaxel minimized the tumor volume at drug doses as small as $5 \mathrm{mg} / \mathrm{kg}$. The side effects were lower than those during traditional therapy [48].

Doxorubicin was attached to the nanocarrier using the amide bond in $[166,176]$. Lai et al. [177] discussed the bioconjugation of drug to the dendrimer. The efficiency of novel system was examined against gingival cancer cell line Ca9-22. The cytotoxicity was improved comparing with free drug.

5.2.4. Disulfide Group. Disulfide bond between drug and nanocarrier is created in order to obtain the systems for selective delivery and accumulation of and large amount of drugs. Most of the drugs and nanocarriers do not possess thiol groups in the structure and thus the method requests preliminary modification. The chemotherapeutic structures possess usually amine and carboxylic groups and these groups may be linked with peptides, the source of the thiol groups. This makes the formation of disulfide bond possible [164].

Paclitaxel bonded by disulfide coupling to SWCNTs was used against leukemia line L1210FR. The disulfide linkage was attached in C-2' position of the drug. SWCNTs were modified by oxidation, conjugation of amide fragments, and functionalization by amine groups. Biotin was used as the targeted ligand. The new DDS successfully destroyed cancer cells. The chemotherapeutic agent was released when the PTX-SWCNTs were entrapped inside cancer cells [178].

5.2.5. Adsorption. Chemotherapeutic agents can be accumulated on the external surface of nanocarriers also by physical adsorption (e.g., by electrostatic interactions between nanocarrier surface and biomolecule) [179-190].

Kataoka et al. [180] described physical adsorption of doxorubicin on the surface of micellar nanocarrier (poly(ethylene glycol)-poly(b-benzyl-Laspartate)) (PEG-PBLA). The drug-nanocarrier system was formed by the $\pi-\pi$ interactions between anthracycline groups of DOX and benzyl residues of PBLA segments. Drug on the nanocarrier revealed greater activity comparing with the free drug. Micelles allowed 
longer circulation time of doxorubicin in the blood. Greish et al. [181] proved 13-time higher drug concentration inside tumor S-180 when the doxorubicin was loaded on the surface of polymeric micelle. The new system showed better therapeutic properties and lower side effects than what is observed in traditional therapy $[180,181]$.

Another study [182] reported the noncovalent attachment of doxorubicin to carbon nanotubes. MWCNTs were covered by triblock copolymer Pluronic F127. Authors proved the rise of DOX cytotoxicity against breast cancer cells line MCF7 in comparison to standard therapy. The use of MWCNTs as drug nanocarrier improved the activity of drug and its uptake by cancer cells. The efficiency of this MWCNTsDOX complex was determined by effective release of drug in suitable time and place [182]. Heister et al. [183] presented a noncovalent complex of doxorubicin with single-walled carbon nanotubes. As targeted, ligand monoclonal antibody was used. Authors proved that SWCNTs can be applied in order to improve the DDS. High activity of DOX adsorbed on the surface of oxidized carbon nanotubes or modified by polycarbohydrate, PEG, PEG44-PPS20 copolymer, and poly(acrylic acid) was proved by Wang's [191], Zhang's [184], Liu’s [185], Di Crescenzo's [186], and Lu's [101] groups, respectively.

Paclitaxel can be adsorbed on the modified surface of nanocarrier. Tian et al. [187] adsorbed PTX on carbon nanotubes by $\pi-\pi$ interactions. MWCNTs were covered with polyethyleneimine. Next the reaction was performed with folic acid as targeted ligand. Drug showed the rise of solubility and was selectively taken up by cancer cells. The activity in vitro of paclitaxel loaded on the surface of carbon nanotubes was greater in comparison to free drug used during traditional therapy [187].

Adsorption of platinum complex is determined by the stability of nitrogen ligand and the mobility of chloride ion. The positively charged platinum aqua-complex is strongly adsorbed on nanocarriers. Adsorption of cisplatin is determined by electrostatic interactions [188]. Barroug et al. [189] studied cisplatin adsorption on nanocarrier and showed the rise of drug adsorption and the rate of drug release with the rise in temperature. The cytotoxicity was tested in vitro against K8 clonal murine osteosarcoma cell line. Electrostatic interactions between cisplatin and the surface of nanoparticle did not change the drug activity. Cisplatin analogue, that is, carboplatin, was adsorbed on the surface of carbon nanotubes by Arlt et al. [190]. Adsorbed drug stopped the growth of tumor, and the efficiency of loaded chemotherapeutic agent was greater than observed for free carboplatin.

Bonding by electrostatic interactions as the method of the drug and nanocarrier bioconjugation is lees popular in comparison to covalent linking. The covalent couplings are preferred due to the possibility of controlling the drug amount and the orientation during synthesis [179].

We described only selected drug accumulation possibilities. Ionic drug complexes for example cisplatin with nanoparticles based on hyaluronic acid [192] or doxorubicin with ionic complex [193] are also discussed in the literature. The covalent functionalization by 1,3-dipolar cycloaddition is also widely used for the bioconjugation of drugs and nanocarrier. Terminal amine groups on the nanocarrier surface are ideal and reactive centers for the coupling of biomolecules [45]. Pastorin et al. [194] discussed the functionalization of MWCNTs via 1,3-dipolar cycloaddition. Active groups formed on the surface of carbon nanotubes were attached with active carboxylic fragments of drug, methotrexate. This coupling minimized the intracellular uptake barrier and caused the increase in the efficiency.

Next interesting attempt is the attachment of two anticancer drugs to one nanocarrier. Few nanocarriers (e.g., polymeric nanoparticle and liposomes) have characteristic properties which are essential during the coupling of different chemotherapeutic agents. Drugs attached together should be easily hydrolyzed, because they are independently aggregated inside cancer cells [20]. This methodology minimizes the drug resistance of cancer cells and releases essential drug dose [195].

Aryal et al. [20] showed the accumulation of paclitaxel and gemcitabine hydrochloride on the nanoparticle surface. The cytotoxicity of drugs comparing with their free analogs was greater against human pancreatic cancer cells XPA3 [20]. Similarly, Zhang et al. presented the linking of doxorubicin with docetaxel [196].

\section{Summary}

Synthesis of a novel DDS, by application of nanomedicine recipes, is very important in anticancer therapy. Application of nanocarriers can improve the activity of drugs. Nanocarriers isolate drug molecules from biological environment and consequently minimize the enzymatic degradation of chemotherapeutic agent [125]. Additionally, nanocarriers cause the rise in solubility and prolong the time of distribution in the blood. Those novel systems break the biological barriers, for example, blood brain. As a consequence, a drug is delivered into the targeted cells, hardly available during standard chemotherapy. The new DDS as targeted ligandnanocarrier drug fulfills all requirements of the effective and safe anticancer therapy: (i) adequate concentration, (ii) effective dose, and (iii) high cytotoxicity of chemotherapeutic agents [195]. The delivery of drug by the application of nanocarriers opens the way to the treatment of diseases showing so-called multidrug resistance [122]. Drugs can be aggregated on the external or internal surface of a nanocarrier. Suitable modification of nanocarrier facilitates the chemical bioconjugation of drug or targeted ligand via the formation the functionalities as amide, ester, disulfide or acetyl-hydrazone groups. The most important factor is the structure of drug or ligand determining the type of reaction.

The results of recent in vitro and in vivo studies [195198] suggest that described novel DDS should be more effective and successful against cancer cells in comparison to traditional chemotherapy. Application of nanovehicles in targeted active treatment seems to be very promising direction in current material science and medicine.

\section{Abbreviations}

BSA: Bovine serum albumin

CC: Click chemistry 


\begin{tabular}{|c|c|}
\hline CNT: & Carbon nanotube \\
\hline DA: & Diels-Alder reaction \\
\hline DDP: & Cisplatin \\
\hline DDS: & Drug delivery dystem \\
\hline DMAP: & N,N-Dimethylaminopyridine \\
\hline DMF: & Dimethylformamide \\
\hline DOX: & Doxorubicin \\
\hline DSP: & Dithiobis(succinimidyl propionate) \\
\hline DSPE: & Phosphatidylethanolamine \\
\hline DTT: & Dithiothreitol \\
\hline EDC: & $\begin{array}{l}\text { 1-Ethyl-3-(3-dimethylaminopropyl) } \\
\text { carbodiimide }\end{array}$ \\
\hline EDX: & Energy-dispersive X-ray spectroscopy \\
\hline EGF: & Epidermal growth factor \\
\hline EPR: & Enhanced permeability and retention effect \\
\hline Fmoc-Osu: & $\begin{array}{l}\text { 9-Fluorenylmethoxycarbonyl-N- } \\
\text { hydroxysuccinimide }\end{array}$ \\
\hline FTIR: & Fourier transform infrared spectroscopy \\
\hline HR-TEM: & $\begin{array}{l}\text { High-resolution transmission electron micro } \\
\text { scopy }\end{array}$ \\
\hline MWCNT: & Multiwalled carbon nanotube \\
\hline NHS: & N-Hydroxysuccinimide \\
\hline PAGE: & Poly(allyl glycidyl ether) \\
\hline PAMAM: & Polyamidoamine dendrimer \\
\hline PCL: & Poly( $\varepsilon$-caprolactone $)$ \\
\hline PBLA: & Poly( $\beta$-benzyl-L-aspartate $)$ \\
\hline PDP: & Pyridyldithiopropionate \\
\hline PDP-PE: & $\begin{array}{l}\mathrm{N} \text {-[3-(2-Pyridyldithio)propionyl]phosphati- } \\
\text { dylethanolamine }\end{array}$ \\
\hline PDP-SA: & $\mathrm{N}$-[3-(2-Pyridylthio)propionyl]-stearylamine \\
\hline PEG: & Polyethylene glycol \\
\hline PEG-b-PCL: & Poly(ethylene glycol)-b-poly( $\varepsilon$-caprolactone) \\
\hline PEO-PAGE: & $\begin{array}{l}\text { Poly(ethylene oxide)-block-poly(allyl glycidy } \\
\text { ether) }\end{array}$ \\
\hline PEG-PBLA: & $\begin{array}{l}\text { Poly(ethylene glycol)-poly(b-benzyl-Laspar } \\
\text { tate) }\end{array}$ \\
\hline PG: & poly(L-glutamic acid) \\
\hline PLA: & Poly(lactic acid) \\
\hline PLGA: & Poly(D,L-lactic-co-glycolic acid) \\
\hline PM: & Polymeric micelle \\
\hline PPS: & Poly(propylene sulfide) \\
\hline PTX: & Paclitaxel \\
\hline PyBroP: & $\begin{array}{l}\text { Bromo-tris-pyrrolidino-phosphonium } \\
\text { hexafluorophosphate }\end{array}$ \\
\hline RT: & Room temperature \\
\hline SATA: & N-Succinimidyl-S-acetylthioacetate \\
\hline SPDP: & $\begin{array}{l}\text { N-Succinimidyl-3- }(2 \\
\text { pyridyldithio)propionate }\end{array}$ \\
\hline SWCNT: & Single-walled carbon nanotube \\
\hline SWNH: & Single-walled carbon nanohorn \\
\hline TEA: & Triethylamine \\
\hline TEM: & Transmission electron microscopy \\
\hline TF: & Transferrin. \\
\hline
\end{tabular}

\section{Conflict of Interests}

The authors declare that there is no conflict of interests regarding the publication of this paper.

\section{Acknowledgment}

The paper was supported by NSC Grant DEC-2011/01/B/ST5/ 01192.

\section{References}

[1] R. A. Freitas Jr., "What is nanomedicine?" Nanomedicine, vol. 1, no. 1, pp. 2-9, 2005.

[2] B. Mishra, B. B. Patel, and S. Tiwari, "Colloidal nanocarriers: a review on formulation technology, types and applications toward targeted drug delivery," Nanomedicine: Nanotechnology, Biology, and Medicine, vol. 6, no. 1, pp. 9-24, 2010.

[3] S. K. Sahoo, S. Parveen, and J. J. Panda, "The present and future of nanotechnology in human health care," Nanomedicine: Nanotechnology, Biology, and Medicine, vol. 3, no. 1, pp. 20-31, 2007.

[4] R. A. Freitas Jr., "What is nanomedicine?" Nanomedicine: Nanotechnology, Biology, and Medicine, vol. 1, no. 1, pp. 2-9, 2005.

[5] K. Kostarelos, "The emergence of nanomedicine: a field in the making," Nanomedicine, vol. 1, no. 1, pp. 1-3, 2006.

[6] C. Wang, S. Ravi, G. V. Martinez et al., "Dual-purpose magnetic micelles for MRI and gene delivery," Journal of Controlled Release, vol. 163, no. 1, pp. 82-92, 2012.

[7] R. G. Mendes, A. Bachmatiuk, B. Büchner, G. Cuniberti, and M. H. Rümmeli, "Carbon nanostructures as multi-functional drug delivery platforms," Journal of Materials Chemistry B, vol. 1, no. 4, pp. 401-428, 2013.

[8] C. Wei, "The valuable and significant role of Nanomedicine," Nanomedicine: Nanotechnology, Biology, and Medicine, vol. 1, no. 4, p. 285, 2005.

[9] O. M. Koo, I. Rubinstein, and H. Onyuksel, "Role of nanotechnology in targeted drug delivery and imaging: a concise review," Nanomedicine, vol. 1, no. 3, pp. 193-212, 2005.

[10] T. Flynn and C. Wei, "The pathway to commercialization for nanomedicine," Nanomedicine: Nanotechnology, Biology, and Medicine, vol. 1, no. 1, pp. 47-51, 2005.

[11] N. Bertrand, J. Wu, X. Xu, N. Kamaly, and O. C. Farokhzad, "Cancer nanotechnology: the impact of passive and active targeting in the era of modern cancer biology," Advanced Drug Delivery Reviews, vol. 66, pp. 2-25, 2014.

[12] Y. Liu, H. Miyoshi, and M. Nakamura, "Nanomedicine for drug delivery and imaging: a promising avenue for cancer therapy and diagnosis using targeted functional nanoparticles," International Journal of Cancer, vol. 120, no. 12, pp. 2527-2537, 2007.

[13] N. K. Mehra, V. Mishra, and N. K. Jain, "A review of ligand tethered surface engineered carbon nanotubes," Biomaterials, vol. 35, no. 4, pp. 1267-1283, 2014.

[14] V. P. Torchilin, "Multifunctional nanocarriers," Advanced Drug Delivery Reviews, vol. 58, no. 14, pp. 1532-1555, 2006.

[15] S. Parveen, R. Misra, and S. K. Sahoo, "Nanoparticles: a boon to drug delivery, therapeutics, diagnostics and imaging," Nanomedicine: Nanotechnology, Biology, and Medicine, vol. 8, no. 2, pp. 147-166, 2012.

[16] G. Minotti, P. Menna, E. Salvatorelli, G. Cairo, and L. Gianni, "Anthracyclines: molecular advances and pharmacologic developments in antitumor activity and cardiotoxicity," Pharmacological Reviews, vol. 56, no. 2, pp. 185-229, 2004. 
[17] J. Verweij, M. Clavel, and B. Chevalier, "Paclitaxel (Taxol) and docetaxel (Taxotere): not simply two of a kind," Annals of Oncology, vol. 5, no. 6, pp. 495-505, 1994.

[18] C. Samorì, H. Ali-Boucetta, R. Sainz et al., "Enhanced anticancer activity of multi-walled carbon nanotube-methotrexate conjugates using cleavable linkers," Chemical Communications, vol. 46, no. 9, pp. 1494-1496, 2010.

[19] Y. Ren and G. Pastorin, "Incorporation of hexamethylmelamine inside capped carbon nanotubes," Advanced Materials, vol. 20, no. 11, pp. 2031-2036, 2008.

[20] S. Aryal, C.-M. J. Hu, and L. Zhang, "Combinatorial drug conjugation enables nanoparticle dual-drug delivery," Small, vol. 6, no. 13, pp. 1442-1448, 2010.

[21] H. S. Oberoi, N. V. Nukolova, A. V. Kabanov, and T. K. Bronich, "Nanocarriers for delivery of platinum anticancer drugs," Advanced Drug Delivery Reviews, vol. 65, no. 13-14, pp. 1667-1685, 2013.

[22] G. Cirillo, S. Hampel, U. G. Spizzirri, O. I. Parisi, N. Picci, and F. Iemma, "Carbon nanotubes hybrid hydrogels in drug delivery: a perspective review," BioMed Research International, vol. 2014, Article ID 825017, 17 pages, 2014.

[23] M. Zhang and M. Yudasaka, "Potential application of nanocarbons as a drug delivery system," Carbon, vol. 69, p. 642, 2014.

[24] G. A. Hughes, "Nanostructure-mediated drug delivery," Nanomedicine, vol. 1, no. 1, pp. 22-30, 2005.

[25] B. Wilson, M. K. Samanta, K. Santhi, K. P. S. Kumar, M. Ramasamy, and B. Suresh, "Chitosan nanoparticles as a new delivery system for the anti-Alzheimer drug tacrine," Nanomedicine, vol. 6, no. 1, pp. el44-e152, 2010.

[26] K. Y. Kim, "Nanotechnology platforms and physiological challenges for cancer therapeutics," Nanomedicine: Nanotechnology, Biology, and Medicine, vol. 3, no. 2, pp. 103-110, 2007.

[27] R. Chouhan and A. K. Bajpai, "Release dynamics of ciprofloxacin from swellable nanocarriers of poly(2-hydroxyethyl methacrylate): an in vitro study," Nanomedicine, vol. 6, no. 3, pp. 453-462, 2010.

[28] E. Koren, A. Apte, A. Jani, and V. P. Torchilin, "Multifunctional PEGylated 2C5-immunoliposomes containing $\mathrm{pH}$ sensitive bonds and TAT peptide for enhanced tumor cell internalization and cytotoxicity," Journal of Controlled Release, vol. 160, no. 2, pp. 264-273, 2012.

[29] A. Koshkaryev, A. Piroyan, and V. P. Torchilin, "Increased apoptosis in cancer cells in vitro and in vivo by ceramides in transferrin-modified liposomes," Cancer Biology and Therapy, vol. 13, no. 1, pp. 50-60, 2012.

[30] A. Etzerodt, M. B. Maniecki, J. H. Graversen, H. J. Moller, V. P. Torchilin, and S. K. Moestrup, "Efficient intracellular drugtargeting of macrophages using stealth liposomes directed to the hemoglobin scavenger receptor CD163," Journal of Controlled Release, vol. 160, no. 1, pp. 72-80, 2012.

[31] K. W. Kang, M.-K. Chun, O. Kim et al., "Doxorubicin-loaded solid lipid nanoparticles to overcome multidrug resistance in cancer therapy," Nanomedicine: Nanotechnology, Biology, and Medicine, vol. 6, no. 2, pp. 210-213, 2010.

[32] U. Kedar, P. Phutane, S. Shidhaye, and V. Kadam, "Advances in polymeric micelles for drug delivery and tumor targeting," Nanomedicine: Nanotechnology, Biology, and Medicine, vol. 6, no. 6, pp. 714-729, 2010.

[33] P. Arukuusk, L. Pärnaste, N. Oskolkov et al., "New generation of efficient peptide-based vectors, NickFects, for the delivery of nucleic acids," Biochimica et Biophysica Acta, vol. 1828, no. 5, pp. 1365-1373, 2013.
[34] Y. Wu, P. Sadatmousavi, R. Wang, S. Lu, Y.-F. Yuan, and P. Chen, "Self-assembling peptide-based nanoparticles enhance anticancer effect of ellipticine in vitro and in vivo," International Journal of Nanomedicine, vol. 7, pp. 3221-3233, 2012.

[35] L. Crombez, M. C. Morris, S. Deshayes, F. Heitz, and G. Divita, "Peptide-based nanoparticle for ex vivo and in vivo dug delivery," Current Pharmaceutical Design, vol. 14, no. 34, pp. 3656-3665, 2008.

[36] A. N. Shirazi, R. Tiwari, B. S. Chhikara, D. Mandal, and K. Parang, "Design and biological evaluation of cell-penetrating peptide-doxorubicin conjugates as prodrugs," Molecular Pharmaceutics, vol. 10, no. 2, pp. 488-499, 2013.

[37] S. Deshayes, K. Konate, A. Rydström et al., "Self-assembling peptide-based nanoparticles for siRNA delivery in primary cell lines," Small, vol. 8, no. 14, pp. 2184-2188, 2012.

[38] K. K. Hou, H. Pan, G. M. Lanza, and S. A. Wickline, "Melittin derived peptides for nanoparticle based siRNA transfection," Biomaterials, vol. 34, no. 12, pp. 3110-3119, 2013.

[39] C. P. Reis, R. J. Neufeld, A. J. Ribeiro, and F. Veiga, "Nanoencapsulation I. Methods for preparation of drug-loaded polymeric nanoparticles," Nanomedicine, vol. 2, no. 1, pp. 8-21, 2006.

[40] N. Mody, R. K. Tekade, N. K. Mehra, P. Chopdey, and N. K. Jain, "Dendrimer, liposomes, carbon nanotubes and PLGA nanoparticles: one platform assessment of drug delivery potential," AAPS PharmSciTech, vol. 15, no. 2, pp. 388-399, 2014.

[41] U. Gupta, H. B. Agashe, A. Asthana, and N. K. Jain, "Dendrimers: novel polymeric nanoarchitectures for solubility enhancement," Nanomedicine, vol. 2, no. 2, pp. 66-73, 2006.

[42] S. Wen, H. Liu, H. Cai, M. Shen, and X. Shi, "Targeted and $\mathrm{pH}$-responsive delivery of doxorubicin to cancer cells using multifunctional dendrimer-modified multi-walled carbon nanotubes," Advanced Healthcare Materials, vol. 2, no. 9, pp. 12671276, 2013.

[43] M. Foldvari and M. Bagonluri, "Carbon nanotubes as functional excipients for nanomedicines: II. Drug delivery and biocompatibility issues," Nanomedicine: Nanotechnology, Biology, and Medicine, vol. 4, no. 3, pp. 183-200, 2008.

[44] A. Shahi, "Carbon nanotubes: a noval carrier system for drug delivery and cancer therapy," International Journal of Pharma and Bio Sciences, vol. 5, no. 3, pp. 298-306, 2014.

[45] C. P. Firme III and P. R. Bandaru, "Toxicity issues in the application of carbon nanotubes to biological systems," Nanomedicine: Nanotechnology, Biology, and Medicine, vol. 6, no. 2, pp. 245256, 2010.

[46] J. Y. Hwang, U. S. Shin, W. C. Jang, J. K. Hyun, I. B. Wall, and H. W. Kim, "Biofunctionalized carbon nanotubes in neural regeneration: a mini-review," Nanoscale, vol. 5, no. 2, pp. 487497, 2013.

[47] H. J. Zhang, C. Chen, L. Hou et al., "Targeting and hyperthermia of doxorubicin by the delivery of single-walled carbon nanotubes to EC-109 cells," Journal of Drug Targeting, vol. 21, no. 3, pp. 312-319, 2013.

[48] Z. Liu, K. Chen, C. Davis et al., "Drug delivery with carbon nanotubes for in vivo cancer treatment," Cancer Research, vol. 68, no. 16, pp. 6652-6660, 2008.

[49] Z. Liu, A. C. Fan, K. Rakhra et al., "Supramolecular stacking of doxorubicin on carbon nanotubes for in vivo cancer therapy," Angewandte Chemie, vol. 48, no. 41, pp. 7668-7672, 2009.

[50] N. W. S. Kam and H. Dai, "Single walled carbon nanotubes for transport and delivery of biological cargos," Physica Status Solidi $B$, vol. 243, no. 13, pp. 3561-3566, 2006. 
[51] N. W. S. Kam, M. O’Connell, J. A. Wisdom, and H. Dai, “Carbon nanotubes as multifunctional biological transporters and nearinfrared agents for selective cancer cell destruction," Proceedings of the National Academy of Sciences of the United States of America, vol. 102, no. 33, pp. 11600-11605, 2005.

[52] N. W. S. Kam, T. C. Jessop, P. A. Wender, and H. Dai, "Nanotube molecular transporters: internalization of carbon nanotube-protein conjugates into mammalian cells," Journal of the American Chemical Society, vol. 126, no. 22, pp. 6850-6851, 2004.

[53] Z. Liu, S. Tabakman, K. Welsher, and H. Dai, "Carbon nanotubes in biology and medicine: in vitro and in vivo detection, imaging and drug delivery," Nano Research, vol. 2, no. 2, pp. 85120, 2009.

[54] Z. Liu, S. M. Tabakman, Z. Chen, and H. Dai, "Preparation of carbon nanotube bioconjugates for biomedical applications," Nature Protocols, vol. 4, no. 9, pp. 1372-1382, 2009.

[55] A. K. Jain, V. Dubey, N. K. Mehra et al., "Carbohydrateconjugated multiwalled carbon nanotubes: development and characterization," Nanomedicine: Nanotechnology, Biology, and Medicine, vol. 5, no. 4, pp. 432-442, 2009.

[56] X. Ma, C. Shu, J. Guo et al., "Targeted cancer therapy based on single-wall carbon nanohorns with doxorubicin in vitro and in vivo," Journal of Nanoparticle Research, vol. 16, no. 7, article 2497, 2014.

[57] S. Han, T. Xia, Q. Li, J. Guo, and P. Lu, "Application of functional quantum dots in cancer diagnosis and therapy: a review," Chinese Journal of Biotechnology, vol. 29, no. 1, pp. 10-20, 2013.

[58] R. Lehner, X. Wang, S. Marsch, and P. Hunziker, "Intelligent nanomaterials for medicine: carrier platforms and targeting strategies in the context of clinical application," Nanomedicine, vol. 9, no. 6, pp. 742-757, 2013.

[59] H. Hosseinzadeh, F. Atyabi, R. Dinarvand, and S. N. Ostad, "Chitosan-Pluronic nanoparticles as oral delivery of anticancer gemcitabine: preparation and in vitro study," International Journal of Nanomedicine, vol. 7, pp. 1851-1863, 2012.

[60] S. Danson, D. Ferry, V. Alakhov et al., "Phase I dose escalation and pharmacokinetic study of pluronic polymer-bound doxorubicin (SP1049C) in patients with advanced cancer," British Journal of Cancer, vol. 90, no. 11, pp. 2085-2091, 2004.

[61] W. X. Mai and H. Meng, "Mesoporous silica nanoparticles: a multifunctional nano therapeutic system," Integrative Biology, vol. 5, no. 1, pp. 19-28, 2013.

[62] Q. Zhang, F. Liu, K. T. Nguyen et al., "Multifunctional mesoporous silica nanoparticles for cancer-targeted and controlled drug delivery," Advanced Functional Materials, vol. 22, no. 24, pp. 5144-5156, 2012.

[63] W. He, Y. Lu, J. Qi, L. Chen, F. Hu, and W. Wu, "Nanoemulsiontemplated shell-crosslinked nanocapsules as drug delivery systems," International Journal of Pharmaceutics, vol. 445, no. 1-2, pp. 69-78, 2013.

[64] B. Brime, P. Frutos, P. Bringas, A. Nieto, M. P. Ballesteros, and G. Frutos, "Comparative pharmacokinetics and safety of a novel lyophilized amphotericin B lecithin-based oil-water microemulsion and amphotericin B deoxycholate in animal models," Journal of Antimicrobial Chemotherapy, vol. 52, no. 1, pp. 103-109, 2003.

[65] E. Che, X. Zheng, C. Sun, D. Chang, T. Jiang, and S. Wang, "Drug nanocrystals: a state of the art formulation strategy for preparing the poorly water-soluble drugs," Asian Journal of Pharmaceutical Sciences, vol. 7, no. 2, pp. 85-95, 2012.
[66] O. Kayser, C. Olbrich, V. Yardley, A. F. Kiderlen, and S. L. Croft, "Formulation of amphotericin B as nanosuspension for oral administration," International Journal of Pharmaceutics, vol. 254, no. 1, pp. 73-75, 2003.

[67] I. Roy, T. Y. Ohulchanskyy, H. E. Pudavar et al., "Ceramicbased nanoparticles entrapping water-insoluble photosensitizing anticancer drugs: a novel drug-carrier system for photodynamic therapy," Journal of the American Chemical Society, vol. 125, no. 26, pp. 7860-7865, 2003.

[68] I. Altintas, R. Heukers, R. van der Meel et al., "Nanobodyalbumin nanoparticles (NANAPs) for the delivery of a multikinase inhibitor 17864 to EGFR overexpressing tumor cells," Journal of Controlled Release, vol. 165, no. 2, pp. 110-118, 2013.

[69] H. Wartlick, B. Spänkuch-Schmitt, K. Strebhardt, J. Kreuter, and K. Langer, "Tumour cell delivery of antisense oligonuclceotides by human serum albumin nanoparticles," Journal of Controlled Release, vol. 96, no. 3, pp. 483-495, 2004.

[70] W. Wu, J. Shen, Z. Gai, K. Hong, P. Banerjee, and S. Zhou, "Multi-functional core-shell hybrid nanogels for $\mathrm{pH}$-dependent magnetic manipulation, fluorescent $\mathrm{pH}$-sensing, and drug delivery," Biomaterials, vol. 32, no. 36, pp. 9876-9887, 2011.

[71] S. V. Vinogradov, E. V. Batrakova, and A. V. Kabanov, "Nanogels for oligonucleotide delivery to the brain," Bioconjugate Chemistry, vol. 15, no. 1, pp. 50-60, 2004.

[72] S. Y. Wang, M. C. Liu, and K. A. Kang, "Magnetic nanoparticles and thermally responsive polymer for targeted hyperthermia and sustained anti-cancer drug delivery," Advances in Experimental Medicine and Biology, vol. 765, pp. 315-321, 2013.

[73] B. A. Moffat, G. Ramachandra Ready, P. McConville et al., "A novel polyacrylamide magnetic nanoparticle contrast agent for molecular imaging using MRI," Molecular Imaging, vol. 2, no. 4, pp. 324-332, 2003.

[74] D. M. Yuan, Y. L. Lv, Y. W. Yao et al., "Efficacy and safety of Abraxane in treatment of progressive and recurrent non-small cell lung cancer patients: a retrospective clinical study," Thoracic Cancer, vol. 3, no. 4, pp. 341-347, 2012.

[75] Z. Yan, L. Xia, H. Qiu, P. Chen, and B. Zhang, "Short-term outcomes of albumin-bound paclitaxel (abraxane)-containing chemotherapy in patients with advanced gastric cancer: a report of 14 cases," The Chinese-German Journal of Clinical Oncology, vol. 12, no. 1, pp. 30-34, 2013.

[76] P. K. Paik, L. P. James, G. J. Riely et al., "A phase 2 study of weekly albumin-bound paclitaxel (Abraxane) given as a twohour infusion," Cancer Chemotherapy and Pharmacology, vol. 68, no. 5, pp. 1331-1337, 2011.

[77] G. H. Hermanson, Bioconjugate Techniques, Academic Press, San Diego, Calif, USA, 2nd edition, 2008.

[78] L. Nobs, F. Buchegger, R. Gurny, and E. Allémann, "Current methods for attaching targeting ligands to liposomes and nanoparticles," Journal of Pharmaceutical Sciences, vol. 93, no. 8, pp. 1980-1992, 2004.

[79] P. Kocbek, N. Obermajer, M. Cegnar, J. Kos, and J. Kristl, “Targeting cancer cells using PLGA nanoparticles surface modified with monoclonal antibody," Journal of Controlled Release, vol. 120, no. 1-2, pp. 18-26, 2007.

[80] O. Ishida, K. Maruyama, H. Tanahashi et al., "Liposomes bearing polyethyleneglycol-coupled transferrin with intracellular targeting property to the solid tumors in vivo," Pharmaceutical Research, vol. 18, no. 7, pp. 1042-1048, 2001.

[81] K. Maruyama, T. Takizawa, T. Yuda, S. J. Kennel, L. Huang, and M. Iwatsuru, "Targetability of novel immunoliposomes 
modified with amphipathic poly(ethylene glycol) s conjugated at their distal terminals to monoclonal antibodies," Biochimica et Biophysica Acta, vol. 1234, no. 1, pp. 74-80, 1995.

[82] K. Maruyama, T. Takizawa, N. Takahashi, T. Tagawa, K. Nagaike, and M. Iwatsuru, "Targeting efficiency of PEGimmunoliposome-conjugated antibodies at PEG terminals," Advanced Drug Delivery Reviews, vol. 24, no. 2-3, pp. 235-242, 1997.

[83] G. Blume, G. Cevc, M. D. J. A. Crommelin, I. A. J. M. Bakker-Woudenberg, C. Kluft, and G. Storm, "Specific targeting with poly(ethylene glycol)-modified liposomes: coupling of homing devices to the ends of the polymeric chains combines effective target binding with long circulation times," Biochimica et Biophysica Acta-Biomembranes, vol. 1149, no. 1, pp. 180-184, 1993.

[84] F. Zeng, H. Lee, and C. Allen, "Epidermal growth factorconjugated poly(ethylene glycol)-block- $\operatorname{poly}(\delta$-valerolactone $)$ copolymer micelles for targeted delivery of chemotherapeutics," Bioconjugate Chemistry, vol. 17, no. 2, pp. 399-409, 2006.

[85] Z. Ou, B. Wu, D. Xing, F. Zhou, H. Wang, and Y. Tang, "Functional single-walled carbon nanotubes based on an integrin $\alpha_{v} \beta_{3}$ monoclonal antibody for highly efficient cancer cell targeting," Nanotechnology, vol. 20, no. 10, Article ID 105102, 2009.

[86] B. Zhang, Q. Chen, H. Tang et al., "Characterization of and biomolecule immobilization on the biocompatible multi-walled carbon nanotubes generated by functionalization with polyamidoamine dendrimers," Colloids and Surfaces B: Biointerfaces, vol. 80, no. 1, pp. 18-25, 2010.

[87] T. Dvir, M. Bauer, A. Schroeder et al., "Nanoparticles targeting the infarcted heart," Nano Letters, vol. 11, no. 10, pp. 4411-4414, 2011.

[88] H.-T. Chou, T.-P. Wang, C.-Y. Lee, N.-H. Tai, and H.-Y. Chang, "Photothermal effects of multi-walled carbon nanotubes on the viability of BT-474 cancer cells," Materials Science and Engineering C, vol. 33, no. 2, pp. 989-995, 2013.

[89] S.-J. Chiu, N. T. Ueno, and R. J. Lee, “Tumor-targeted gene delivery via anti-HER2 antibody (trastuzumab, Herceptin) conjugated polyethylenimine," Journal of Controlled Release, vol. 97, no. 2, pp. 357-369, 2004.

[90] F. J. Martin and D. Papahadjopoulos, "Irreversible coupling of immunoglobulin fragments to preformed vesicles. An improved method for liposome targeting," The Journal of Biological Chemistry, vol. 257, no. 1, pp. 286-288, 1982.

[91] D. Kirpotin, J. W. Park, K. Hong et al., "Sterically stabilized antiHER2 immunoliposomes: design and targeting to human breast cancer cells in vitro," Biochemistry, vol. 36, no. 1, pp. 66-75, 1997.

[92] T. M. Allen, E. Brandeis, C. B. Hansen, G. Y. Kao, and S. Zalipsky, "A new strategy for attachment of antibodies to sterically stabilized liposomes resulting in efficient targeting to cancer cells," Biochimica et Biophysica Acta - Biomembranes, vol. 1237, no. 2, pp. 99-108, 1995.

[93] C. B. Hansen, G. Y. Kao, E. H. Moase, S. Zalipsky, and T. M. Allen, "Attachment of antibodies to sterically stabilized liposomes: evaluation, comparison and optimization of coupling procedures," Biochimica et Biophysica Acta-Biomembranes, vol. 1239, no. 2, pp. 133-144, 1995.

[94] S. Zalipsky, M. Newman, P. Bhagya, and C. M. Woodle, "Model ligands linked to the polymer-chains on liposomal surfaces: application of a new functionalized polyethylene glycol-lipid conjugate," Polymeric Materials: Science and Engineering, vol. 67, pp. 519-520, 1993.
[95] J. W. Park, K. Hong, P. Carter et al., "Development of antip185HER2 immunoliposomes for cancer therapy," Proceedings of the National Academy of Sciences of the United States of America, vol. 92, no. 5, pp. 1327-1331, 1995.

[96] J. Ren, S. Shen, D. Wang et al., "The targeted delivery of anticancer drugs to brain glioma by PEGylated oxidized multiwalled carbon nanotubes modified with angiopep-2," Biomaterials, vol. 33, no. 11, pp. 3324-3333, 2012.

[97] M. G. Anhorn, S. Wagner, J. Kreuter, K. Langer, and H. Von Briesen, "Specific targeting of HER2 overexpressing breast cancer cells with doxorubicin-loaded trastuzumab-modified human serum albumin nanoparticles," Bioconjugate Chemistry, vol. 19, no. 12, pp. 2321-2331, 2008.

[98] M. E. Gindy, S. Ji, T. R. Hoye, A. Z. Panagiotopoulos, and R. K. Prud'homme, "Preparation of poly(ethylene glycol) protected nanoparticles with variable bioconjugate ligand density," Biomacromolecules, vol. 9, no. 10, pp. 2705-2711, 2008.

[99] O. C. Farokhzad, S. Jon, A. Khademhosseini, T.-N. T. Tran, D. A. LaVan, and R. Langer, "Nanoparticle-aptamer bioconjugates: a new approach for targeting prostate cancer cells," Cancer Research, vol. 64, no. 21, pp. 7668-7672, 2004.

[100] Z. Xiao, E. Levy-Nissenbaum, F. Alexis et al., "Engineering of targeted nanoparticles for cancer therapy using internalizing aptamers isolated by cell-uptake selection," ACS Nano, vol. 61, no. 1, pp. 696-704, 2012.

[101] Y. J. Lu, K. C. Wei, C. C. M. Ma, S. Y. Yang, and J. P. Chen, "Dual targeted delivery of doxorubicin to cancer cells using folateconjugated magnetic multi-walled carbon nanotubes," Colloids and Surfaces B: Biointerfaces, vol. 89, no. 1, pp. 1-9, 2012.

[102] F. J. Martin, W. L. Hubbell, and D. Papahadjopoulos, "Immunospecific targeting of liposomes to cells: a novel and efficient method for covalent attachment of Fab' fragments via disulfide bonds," Biochemistry, vol. 20, no. 14, pp. 4229-4238, 1981.

[103] V. O. Ivanov, S. N. Preobrazhensky, V. P. Tsibulsky, V. R. Babaev, V. S. Repin, and V. N. Smirnov, "Liposome uptake by cultured macrophages mediated by modified low-density lipoproteins," Biochimica et Biophysica Acta, vol. 846, no. 1, pp. 76-84, 1985.

[104] M. S. Shaik, N. Kanikkannan, and M. Singh, "Conjugation of anti-My9 antibody to stealth monensin liposomes and the effect of conjugated liposomes on the cytotoxicity of immunotoxin," Journal of Controlled Release, vol. 76, no. 3, pp. 285-295, 2001.

[105] M. Talelli, S. Oliveira, C. J. F. Rijcken et al., "Intrinsically active nanobody-modified polymeric micelles for tumor-targeted combination therapy," Biomaterials, vol. 34 , no. 4, pp. 12551260, 2013.

[106] D. E. L. de Menezes, L. M. Pilarski, and T. M. Allen, "In vitro and in vivo targeting of immunoliposomal doxorubicin to human Bcell lymphoma," Cancer Research, vol. 58, no. 15, pp. 3320-3330, 1998.

[107] M. M. Chua, S. T. Fan, and F. Karush, "Attachment of immunoglobulin to liposomal membrane via protein carbohydrate," Biochimica et Biophysica Acta, vol. 800, no. 3, pp. 291300, 1984.

[108] S. M. Chamow, T. P. Kogan, D. H. Peers, R. C. Hastings, R. A. Byrn, and A. Ashkenazi, "Conjugation of soluble CD4 without loss of biological activity via a novel carbohydrate-directed cross-linking reagent," The Journal of Biological Chemistry, vol. 267, no. 22, pp. 15916-15922, 1992.

[109] J. A. Harding, C. M. Engbers, M. S. Newman, N. I. Goldstein, and S. Zalipsky, "Immunogenicity and pharmacokinetic attributes of poly( ethylene glycol)-grafted immunoliposomes," 
Biochimica et Biophysica Acta-Biomembranes, vol. 1327, no. 2, pp. 181-192, 1997.

[110] M. Shi, J. H. Wosnick, K. Ho, A. Keating, and M. S. Shoichet, "Immuno-polymeric nanoparticles by Diels-Alder chemistry," Angewandte Chemie, vol. 46, no. 32, pp. 6126-6131, 2007.

[111] A. D. de Araújo, J. M. Palomo, J. Cramer et al., "Diels-Alder ligation and surface immobilization of proteins," Angewandte Chemie International Edition, vol. 45, no. 2, pp. 296-301, 2005.

[112] V. Marchan, S. Ortega, D. Pulido, E. Pedroso, and A. Grandas, "Diels-Alder cycloadditions in water for the straightforward preparation of peptide-oligonucleotide conjugates," Nucleic Acids Research, vol. 34, no. 3, p. e24, 2006.

[113] H. C. Kolb, M. G. Finn, and K. B. Sharpless, "Click chemistry: diverse chemical function from a few good reactions," Angewandte Chemie International Edition, vol. 40, no. 11, pp. 20042021, 2001.

[114] C. D. Hein, X.-M. Liu, and D. Wang, "Click chemistry, a powerful tool for pharmaceutical sciences," Pharmaceutical Research, vol. 25, no. 10, pp. 2216-2230, 2008.

[115] B. Jeong, Y. H. Bae, D. S. Lee, and S. W. Kim, "Biodegradable block copolymers as injectable drug-delivery systems," Nature, vol. 388 , no. 6645 , pp. 860-862, 1997.

[116] F. S. Hassane, B. Frisch, and F. Schuber, "Targeted liposomes: convenient coupling of ligands to preformed vesicles using "click chemistry"', Bioconjugate Chemistry, vol. 17, no. 3, pp. 849-854, 2006.

[117] P. De, S. R. Gondi, and B. S. Sumerlin, "Folate-conjugated thermoresponsive block copolymers: highly efficient conjugation and solution self-assembly," Biomacromolecules, vol. 9, no. 3, pp. 1064-1070, 2008.

[118] J. Lu, M. Shi, and M. S. Shoichet, "Click chemistry functionalized polymeric nanoparticles target corneal epithelial cells through RGD-cell surface receptors," Bioconjugate Chemistry, vol. 20, no. 1, pp. 87-94, 2009.

[119] R. R. Sawant and V. P. Torchilin, "Challenges in development of targeted liposomal therapeutics," The AAPS Journal, vol. 14, no. 2, pp. 303-315, 2012.

[120] H. Zhang, Y. Ma, and X.-L. Sun, "Chemically-selective surface glyco-functionalization of liposomes through Staudinger ligation," Chemical Communications, no. 21, pp. 3032-3034, 2009.

[121] M.-Q. Gu, X.-B. Yuan, C.-S. Kang et al., "Surface biofunctionalization of PLA nanoparticles through amphiphilic polysaccharide coating and ligand coupling: evaluation of biofunctionalization and drug releasing behavior," Carbohydrate Polymers, vol. 67, no. 3, pp. 417-426, 2007.

[122] D. Peer, J. M. Karp, S. Hong, O. C. Farokhzad, R. Margalit, and R. Langer, "Nanocarriers as an emerging platform for cancer therapy," Nature Nanotechnology, vol. 2, no. 12, pp. 751-760, 2007.

[123] B. S. Wong, S. L. Yoong, A. Jagusiak et al., "Carbon nanotubes for delivery of small molecule drugs," Advanced Drug Delivery Reviews, vol. 65, no. 15, pp. 1964-2015, 2013.

[124] R. Sinha, G. J. Kim, S. Nie, and D. M. Shin, "Nanotechnology in cancer therapeutics: bioconjugated nanoparticles for drug delivery," Molecular Cancer Therapeutics, vol. 5, no. 8, pp. 19091917, 2006.

[125] V. Torchilin, "Multifunctional and stimuli-sensitive pharmaceutical nanocarriers," European Journal of Pharmaceutics and Biopharmaceutics, vol. 71, no. 3, pp. 431-444, 2009.

[126] D. R. Khan, "The use of nanocarriers for drug delivery in cancer therapy," Journal of Cancer Science and Therapy, vol. 2, no. 3, pp. 58-62, 2010.
[127] H. S. Yoo, K. H. Lee, J. E. Oh, and T. G. Park, "In vitro and in vivo anti-tumor activities of nanoparticles based on doxorubicinPLGA conjugates," Journal of Controlled Release, vol. 68, no. 3, pp. 419-431, 2000.

[128] H. S. Yoo, J. E. Oh, K. H. Lee, and T. G. Park, "Biodegradable nanoparticles containing doxorubicin-PLGA conjugate for sustained release," Pharmaceutical Research, vol. 16, no. 7, pp. 1114$1118,1999$.

[129] T. L. Moore, J. E. Pitzer, R. Podila et al., "Multifunctional polymer-coated carbon nanotubes for safe drug delivery," Particle and Particle Systems Characterization, vol. 30, no. 4, pp. 365373, 2013.

[130] L. Juillerat-Jeanneret, "The targeted delivery of cancer drugs across the blood-brain barrier: chemical modifications of drugs or drug-nanoparticles?" Drug Discovery Today, vol. 13, no. 2324, pp. 1099-1106, 2008.

[131] S. K. Vashist, D. Zheng, K. Al-Rubeaan, J. H. T. Luong, and F.S. Sheu, "Advances in carbon nanotube based electrochemical sensors for bioanalytical applications," Biotechnology Advances, vol. 29, no. 2, pp. 169-188, 2011.

[132] R. Marega and D. Bonifazi, "Filling carbon nanotubes for nanobiotechnological applications," New Journal of Chemistry, vol. 38, no. 1, pp. 22-27, 2014.

[133] M. Yudasaka, K. Ajima, K. Suenaga, T. Ichihashi, A. Hashimoto, and S. Iijima, "Nano-extraction and nano-condensation for $\mathrm{C}_{60}$ incorporation into single-wall carbon nanotubes in liquid phases," Chemical Physics Letters, vol. 380, no. 1-2, pp. 42-46, 2003.

[134] C. Tripisciano, K. Kraemer, A. Taylor, and E. Borowiak-Palen, "Single-wall carbon nanotubes based anticancer drug delivery system," Chemical Physics Letters, vol. 478, no. 4-6, pp. 200-205, 2009.

[135] C. Tripisciano, S. Costa, R. J. Kalenczuk, and E. Borowiak-Palen, "Cisplatin filled multiwalled carbon nanotubes-a novel molecular hybrid of anticancer drug container," European Physical Journal B, vol. 75, no. 2, pp. 141-146, 2010.

[136] J. Li, S. Q. Yap, S. L. Yoong et al., "Carbon nanotube bottles for incorporation, release and enhanced cytotoxic effect of cisplatin," Carbon, vol. 50, no. 4, pp. 1625-1634, 2012.

[137] L. Sui, T. Yang, P. Gao et al., "Incorporation of cisplatin into PEG-wrapped ultrapurified large-inner-diameter MWCNTs for enhanced loading efficiency and release profile," International Journal of Pharmaceutics, vol. 471, no. 1-2, pp. 157-165, 2014.

[138] E. C. Gryparis, M. Hatziapostolou, E. Papadimitriou, and K. Avgoustakis, "Anticancer activity of cisplatin-loaded PLGAmPEG nanoparticles on LNCaP prostate cancer cells," European Journal of Pharmaceutics and Biopharmaceutics, vol. 67, no. 1, pp. 1-8, 2007.

[139] N. Nishiyama and K. Kataoka, "Preparation and characterization of size-controlled polymeric micelle containing cisdichlorodiammineplatinum(II) in the core," Journal of Controlled Release, vol. 74, no. 1-3, pp. 83-94, 2001.

[140] Y. Mizumura, Y. Matsumura, T. Hamaguchi et al., "Cisplatinincorporated polymeric micelles eliminate nephrotoxicity, while maintaining antitumor activity," Japanese Journal of Cancer Research, vol. 92, no. 3, pp. 328-336, 2001.

[141] A. Schroeder, R. Honen, K. Turjeman, A. Gabizon, J. Kost, and Y. Barenholz, "Ultrasound triggered release of cisplatin from liposomes in murine tumors," Journal of Controlled Release, vol. 137, no. 1, pp. 63-68, 2009. 
[142] M. S. Newman, G. T. Colbern, P. K. Working, C. Engbers, and M. A. Amantea, "Comparative pharmacokinetics, tissue distribution, and therapeutic effectiveness of cisplatin encapsulated in long-circulating, pegylated liposomes (SPI-077) in tumorbearing mice," Cancer Chemotherapy and Pharmacology, vol. 43, no. 1, pp. 1-7, 1999.

[143] K. Ajima, M. Yudasaka, T. Murakami, A. Maigné, K. Shiba, and S. Iijima, "Carbon nanohorns as anticancer drug carriers," Molecular Pharmaceutics, vol. 2, no. 6, pp. 475-480, 2005.

[144] K. Ajima, T. Murakami, Y. Mizoguchi et al., "Enhancement of in vivo anticancer effects of cisplatin by incorporation inside single-wall carbon nanohorns," ACS Nano, vol. 2, no. 10, pp. 2057-2064, 2008.

[145] S. Hampel, D. Kunze, D. Haase et al., "Carbon nanotubes filled with a chemotherapeutic agent: a nanocarrier mediates inhibition of tumor cell growth," Nanomedicine, vol. 3, no. 2, pp. 175-182, 2008.

[146] N. Graf, D. R. Bielenberg, N. Kolishetti et al., " $\alpha_{v} \beta_{3}$ integrintargeted PLGA-PEG nanoparticles for enhanced anti-tumor efficacy of a Pt(IV) prodrug," ACS Nano, vol. 6, no. 5, pp. 45304539, 2012.

[147] S. Mitra, U. Gaur, P. C. Ghosh, and A. N. Maitra, "Tumour targeted delivery of encapsulated dextran-doxorubicin conjugate using chitosan nanoparticles as carrier," Journal of Controlled Release, vol. 74, no. 1-3, pp. 317-323, 2001.

[148] F. Lince, S. Bolognesi, B. Stella, D. L. Marchisio, and F. Dosio, "Preparation of polymer nanoparticles loaded with doxorubicin for controlled drug delivery," Chemical Engineering Research and Design, vol. 89, no. 11, pp. 2410-2419, 2011.

[149] H. Park, J. Yang, J. Lee, S. Haam, I.-H. Choi, and K.-H. Yoo, "Multifunctional nanoparticles for combined doxorubicin and photothermal treatments," ACS Nano, vol. 3, no. 10, pp. 29192926, 2009.

[150] Y. Matsumura, M. Gotoh, K. Muro et al., "Phase I and pharmacokinetic study of MCC-465, a doxorubicin (DXR) encapsulated in PEG immunoliposome, in patients with metastatic stomach cancer," Annals of Oncology, vol. 15, no. 3, pp. 517-525, 2004.

[151] A. A. Gabizon, "Pegylated liposomal doxorubicin: metamorphosis of an old drug into a new form of chemotherapy," Cancer Investigation, vol. 19, no. 4, pp. 424-436, 2001.

[152] K. Kataoka, T. Matsumoto, M. Yokoyama et al., "Doxorubicinloaded poly(ethylene glycol)-poly( $\beta$-benzyl-L-aspartate) copolymer micelles: their pharmaceutical characteristics and biological significance," Journal of Controlled Release, vol. 64, no. 1-3, pp. 143-153, 2000.

[153] F. Perche, N. R. Patel, and V. P. Torchilin, "Accumulation and toxicity of antibody-targeted doxorubicin-loaded PEG-PE micelles in ovarian cancer cell spheroid model," Journal of Controlled Release, vol. 164, no. 1, pp. 95-102, 2012.

[154] A. B. Ebrahim Attia, C. Yang, J. P. K. Tan et al., "The effect of kinetic stability on biodistribution and anti-tumor efficacy of drug-loaded biodegradable polymeric micelles," Biomaterials, vol. 34, no. 12, pp. 3132-3140, 2013.

[155] A. Cambón, A. Rey-Rico, D. Mistry et al., "Doxorubicin-loaded micelles of reverse poly(butylene oxide)-poly(ethylene oxide)poly(butylene oxide) block copolymers as efficient 'active' chemotherapeutic agents," International Journal of Pharmaceutics, vol. 445, no. 1-2, pp. 47-57, 2013.

[156] J. M. Koziara, P. R. Lockman, D. D. Allen, and R. J. Mumper, "Paclitaxel nanoparticles for the potential treatment of brain tumors," Journal of Controlled Release, vol. 99, no. 2, pp. 259269, 2004.

[157] G. Ruan and S.-S. Feng, "Preparation and characterization of poly(lactic acid)-poly(ethylene glycol)-poly(lactic acid) (PLAPEG-PLA) microspheres for controlled release of paclitaxel," Biomaterials, vol. 24, no. 27, pp. 5037-5044, 2003.

[158] C. Fonseca, S. Simões, and R. Gaspar, "Paclitaxel-loaded PLGA nanoparticles: preparation, physicochemical characterization and in vitro anti-tumoral activity," Journal of Controlled Release, vol. 83, no. 2, pp. 273-286, 2002.

[159] J. M. Chan, J. W. Rhee, C. L. Drum et al., "In vivo prevention of arterial restenosis with paclitaxel-encapsulated targeted lipidpolymeric nanoparticles," Proceedings of the National Academy of Sciences of the United States of America, vol. 108, no. 48, pp. 19347-19352, 2011.

[160] T. Hamaguchi, K. Kato, H. Yasui et al., "A phase I and pharmacokinetic study of NK105, a paclitaxel-incorporating micellar nanoparticle formulation," British Journal of Cancer, vol. 97, no. 2, pp. 170-176, 2007.

[161] T.-Y. Kim, D.-W. Kim, J.-Y. Chung et al., "Phase I and pharmacokinetic study of Genexol-PM, a Cremophor-free, polymeric micelle-formulated paclitaxel, in patients with advanced malignancies," Clinical Cancer Research, vol. 10, no. 11, pp. 3708-3716, 2004.

[162] F. Wang, Y. Chen, D. Zhang et al., "Folate-mediated targeted and intracellular delivery of paclitaxel using a novel deoxycholic acid-O-carboxymethylated chitosan-folic acid micelles," International Journal of Nanomedicine, vol. 7, pp. 325-337, 2012.

[163] Z. Liu, J. T. Robinson, S. M. Tabakman, K. Yang, and H. Dai, "Carbon materials for drug delivery and cancer therapy," Materials Today, vol. 14, no. 7-8, pp. 316-323, 2011.

[164] K. R. West and S. Otto, "Reversible covalent chemistry in drug delivery," Current Drug Discovery Technologies, vol. 2, no. 3, pp. 123-160, 2005.

[165] C. Li, R. A. Newman, Q.-P. Wu et al., "Biodistribution of paclitaxel and poly(L-glutamic acid)-paclitaxel conjugate in mice with ovarian OCa-1 tumor," Cancer Chemotherapy and Pharmacology, vol. 46, no. 5, pp. 416-422, 2000.

[166] L. Milas, K. A. Mason, N. Hunter, C. Li, and S. Wallace, "Poly(L-glutamic acid)-paclitaxel conjugate is a potent enhancer of tumor radiocurability," International Journal of Radiation Oncology Biology Physics, vol. 55, no. 3, pp. 707-712, 2003.

[167] T. Etrych, T. Mrkvan, P. Chytil, Č. Koňák, B. Ř́hová, and K. Ulbrich, "N-(2-hydroxypropyl)methacrylamide-based polymer conjugates with $\mathrm{pH}$-controlled activation of doxorubicin. I. New synthesis, physicochemical characterization and preliminary biological evaluation," Journal of Applied Polymer Science, vol. 109, no. 5, pp. 3050-3061, 2008.

[168] M. Hrubý, Č. Koňák, and K. Ulbrich, "Polymeric micellar $\mathrm{pH}$-sensitive drug delivery system for doxorubicin," Journal of Controlled Release, vol. 103, no. 1, pp. 137-148, 2005.

[169] D. Vetvicka, M. Hruby, O. Hovorka et al., "Biological evaluation of polymeric micelles with covalently bound doxorubicin," Bioconjugate Chemistry, vol. 20, no. 11, pp. 2090-2097, 2009.

[170] C. Liu, F. Liu, L. Feng, M. Li, J. Zhang, and N. Zhang, "The targeted co-delivery of DNA and doxorubicin to tumor cells via multifunctional PEI-PEG based nanoparticles," Biomaterials, vol. 34, no. 10, pp. 2547-2564, 2013.

[171] H. S. Yoo, E. A. Lee, and T. G. Park, “Doxorubicin-conjugated biodegradable polymeric micelles having acid-cleavable linkages," Journal of Controlled Release, vol. 82, no. 1, pp. 17-27, 2002. 
[172] S. Aryal, C.-M. J. Hu, and L. Zhang, "Polymer-cisplatin conjugate nanoparticles for acid-responsive drug delivery," ACS Nano, vol. 4, no. 1, pp. 251-258, 2010.

[173] E. Tsai, "Dendrimer encapsulated nanoparticles: vehicles for drug delivery, with an emphasis on PAMAM dendrimers," Cosmos, vol. 8, pp. 1-16, 2011.

[174] P. C. A. Rodrigues, K. Scheuermann, C. Stockmar et al., "Synthesis and in vitro efficacy of acid-sensitive poly(ethylene glycol) paclitaxel conjugates," Bioorganic and Medicinal Chemistry Letters, vol. 13, no. 3, pp. 355-360, 2003.

[175] R. P. Feazell, N. Nakayama-Ratchford, H. Dai, and S. J. Lippard, "Soluble single-walled carbon nanotubes as longboat delivery systems for platinum(IV) anticancer drug design," Journal of the American Chemical Society, vol. 129, no. 27, pp. 8438-8439, 2007.

[176] Y.-Z. Zhao, C.-Z. Sun, C.-T. Lu et al., "Characterization and anti-tumor activity of chemical conjugation of doxorubicin in polymeric micelles (DOX-P) in vitro," Cancer Letters, vol. 311, no. 2, pp. 187-194, 2011.

[177] P.-S. Lai, P.-J. Lou, C.-L. Peng et al., "Doxorubicin delivery by polyamidoamine dendrimer conjugation and photochemical internalization for cancer therapy," Journal of Controlled Release, vol. 122, no. 1, pp. 39-46, 2007.

[178] J. Chen, S. Chen, X. Zhao, L. V. Kuznetsova, S. S. Wong, and I. Ojima, "Functionalized single-walled carbon nanotubes as rationally designed vehicles for tumor-targeted drug delivery," Journal of the American Chemical Society, vol. 130, no. 49, pp. 16778-16785, 2008.

[179] L. Niu, L. Meng, and Q. Lu, "Folate-conjugated PEG on single walled carbon nanotubes for targeting delivery of doxorubicin to cancer cells," Macromolecular Bioscience, vol. 13, no. 6, pp. 735-744, 2013.

[180] K. Kataoka, T. Matsumoto, M. Yokoyama et al., "Doxorubicinloaded poly(ethylene glycol)-poly(beta-benzyl-L-aspartate) copolymer micelles: their pharmaceutical characteristics and biological significance," Journal of Controlled Release, vol. 64, no. 1-3, pp. 143-153, 2000.

[181] K. Greish, T. Sawa, J. Fang, T. Akaike, and H. Maeda, "SMAdoxorubicin, a new polymeric micellar drug for effective targeting to solid tumours," Journal of Controlled Release, vol. 97, no. 2, pp. 219-230, 2004.

[182] H. Ali-Boucetta, K. T. Al-Jamal, D. McCarthy, M. Prato, A. Bianco, and K. Kostarelos, "Multiwalled carbon nanotubedoxorubicin supramolecular complexes for cancer therapeutics," Chemical Communications, vol. 8, no. 4, pp. 459-461, 2008.

[183] E. Heister, V. Neves, C. Tîlmaciu et al., “Triple functionalisation of single-walled carbon nanotubes with doxorubicin, a monoclonal antibody, and a fluorescent marker for targeted cancer therapy," Carbon, vol. 47, no. 9, pp. 2152-2160, 2009.

[184] X. Zhang, L. Meng, Q. Lu, Z. Fei, and P. J. Dyson, “Targeted delivery and controlled release of doxorubicin to cancer cells using modified single wall carbon nanotubes," Biomaterials, vol. 30, no. 30, pp. 6041-6047, 2009.

[185] Z. Liu, X. Sun, N. Nakayama-Ratchford, and H. Dai, "Supramolecular chemistry on water-soluble carbon nanotubes for drug loading and delivery," ACS Nano, vol. 1, no. 1, pp. 50-56, 2007.

[186] A. Di Crescenzo, D. Velluto, J. A. Hubbell, and A. Fontana, "Biocompatible dispersions of carbon nanotubes: a potential tool for intracellular transport of anticancer drugs," Nanoscale, vol. 3, no. 3, pp. 925-928, 2011.

[187] Z. Tian, Y. Shi, M. Yin, H. Shen, and N. Jia, "Functionalized multiwalled carbon nanotubes-anticancer drug carriers: synthesis, targeting ability and antitumor activity," Nano Biomedicine and Engineering, vol. 3, no. 3, pp. 157-162, 2011.

[188] A. Duma, M. Prodana, and I. Demetrescu, "Cisplatin functionalization of multiwall carbon nanotubes," UPB Scientific Bulletin, Series B: Chemistry and Materials Science, vol. 76, no. 1, pp. 49-58, 2014.

[189] A. Barroug, L. T. Kuhn, L. C. Gerstenfeld, and M. J. Glimcher, "Interactions of cisplatin with calcium phosphate nanoparticles: in vitro controlled adsorption and release," Journal of Orthopaedic Research, vol. 22, no. 4, pp. 703-708, 2004.

[190] M. Arlt, D. Haase, S. Hampel et al., "Delivery of carboplatin by carbon-based nanocontainers mediates increased cancer cell death," Nanotechnology, vol. 21, no. 33, Article ID 335101, 2010.

[191] Y. Wang, S.-T. Yang, Y. Wang, Y. Liu, and H. Wang, "Adsorption and desorption of doxorubicin on oxidized carbon nanotubes," Colloids and Surfaces B: Biointerfaces, vol. 97, pp. 62-69, 2012.

[192] Y.-I. Jeong, S.-T. Kim, S.-G. Jin et al., "Cisplatin-lncorporated hyaluronic acid nanoparticles based on ion-complex formation," Journal of Pharmaceutical Sciences, vol. 97, no. 3, pp. 12681276, 2008.

[193] T. K. Bronich, A. Nehls, A. Eisenberg, V. A. Kabanov, and A. V. Kabanov, "Novel drug delivery systems based on the complexes of block ionomers and surfactants of opposite charge," Colloids and Surfaces B: Biointerfaces, vol. 16, no. 1-4, pp. 243-251, 1999.

[194] G. Pastorin, W. Wu, S. Wieckowski et al., "Double functionalisation of carbon nanotubes for multimodal drug delivery," Chemical Communications, no. 11, pp. 1182-1184, 2006.

[195] R. Li, R. Wu, L. Zhao, M. Wu, L. Yang, and H. Zou, "Pglycoprotein antibody functionalized carbon nanotube overcomes the multidrug resistance of human leukemia cells," ACS Nano, vol. 4, no. 3, pp. 1399-1408, 2010.

[196] L. Zhang, A. F. Radovic-Moreno, F. Alexis et al., "Co-delivery of hydrophobic and hydrophilic drugsfrom nanoparticle-aptamer bioconjugates," ChemMedChem, vol. 2, no. 9, pp. 1268-1271, 2007.

[197] K. Werengowska-Ciećwierz, M. Wiśniewski, A. P. Terzyk et al., "Nanotube-mediated efficiency of cisplatin anticancer therapy," Carbon, vol. 70, pp. 46-58, 2014.

[198] N. Lodhi, N. K. Mehra, and N. K. Jain, "Development and characterization of dexamethasone mesylate anchored on multi walled carbon nanotubes," Journal of Drug Targeting, vol. 21, no. 1, pp. 67-76, 2013.

[199] J. Shi, Z. Xiao, N. Kamaly, and O. C. Farokhzad, "Self-assembled targeted nanoparticles: evolution of technologies and bench to bedside translation," Accounts of Chemical Research, vol. 44, no. 10, pp. 1123-1134, 2011. 

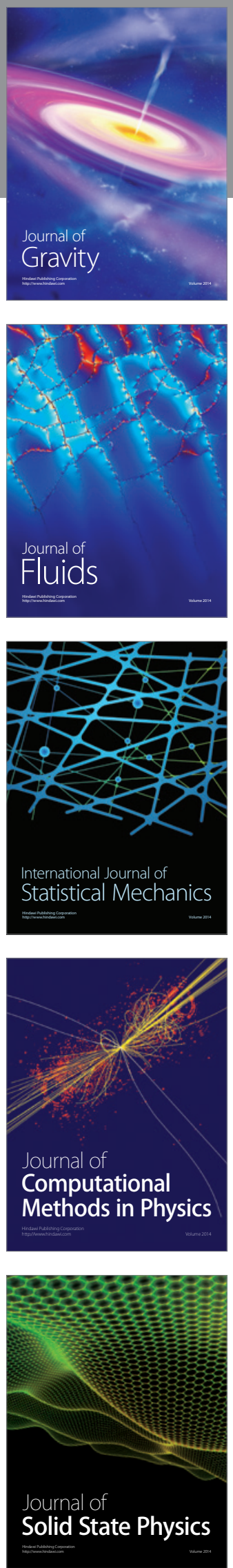

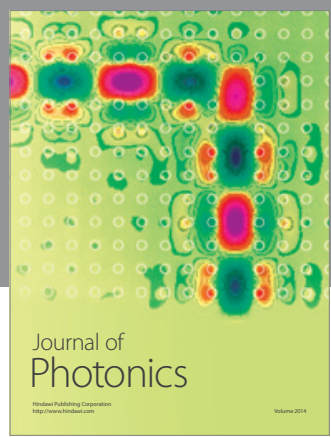

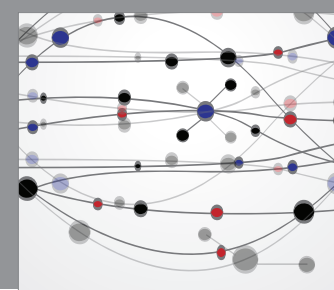

The Scientific World Journal

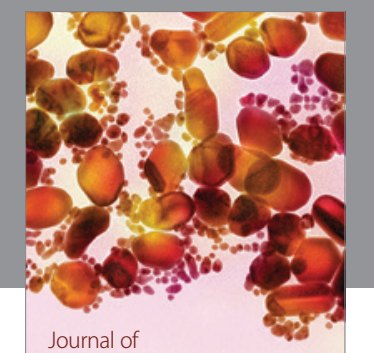

Soft Matter
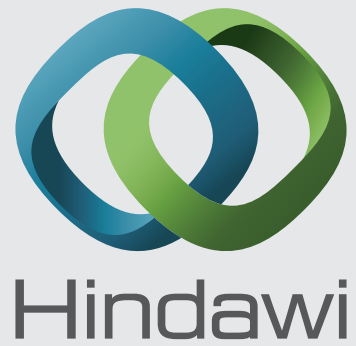

Submit your manuscripts at

http://www.hindawi.com
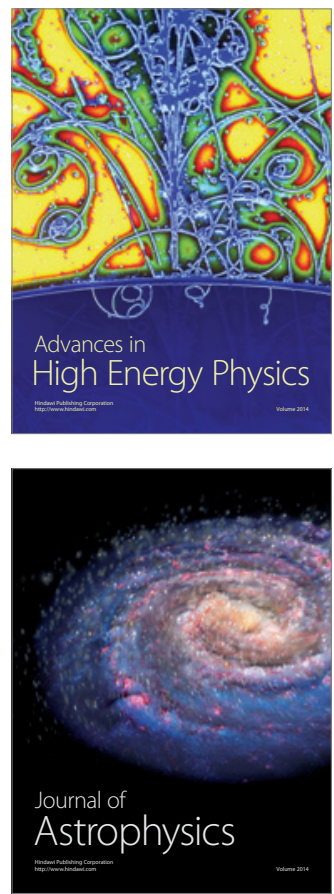
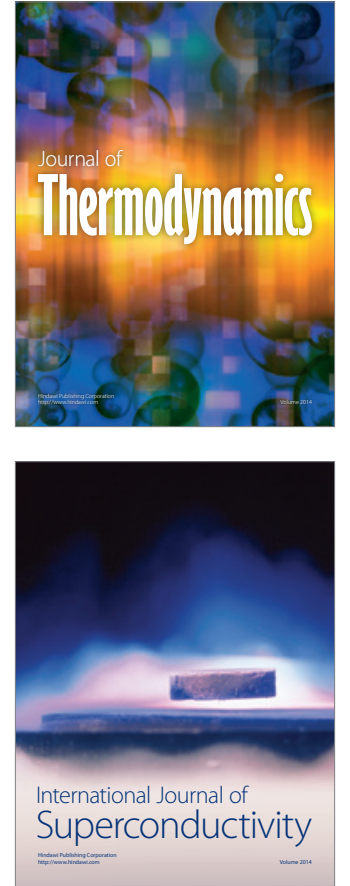
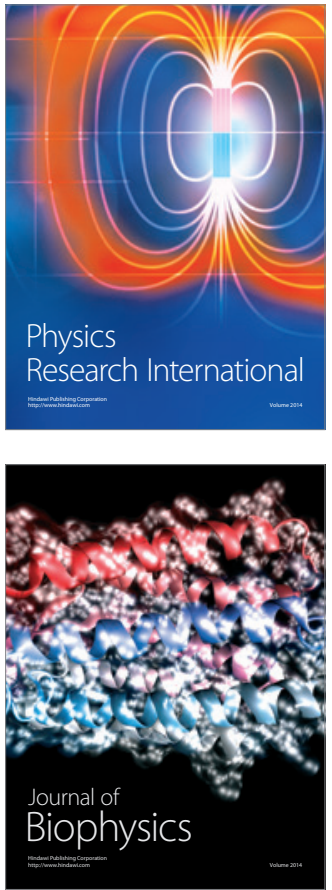
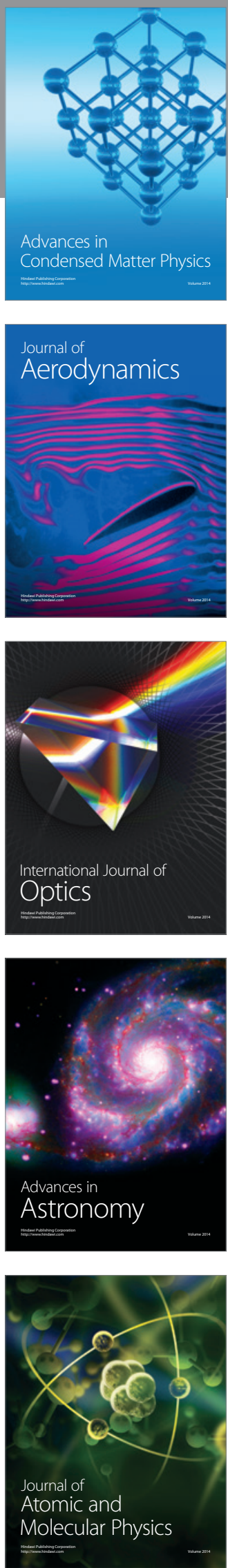